\title{
An Extended VIKOR Method for Multiple Attribute Decision Analysis with Bidimensional Dual Hesitant Fuzzy Information
}

\author{
Min Xue, ${ }^{1,2}$ Xiaoan Tang, ${ }^{1,2}$ and Nanping Feng ${ }^{1,2}$ \\ ${ }^{1}$ School of Management, Hefei University of Technology, P.O. Box 270, Hefei, Anhui 230009, China \\ ${ }^{2}$ Key Laboratory of Process Optimization and Intelligent Decision-Making, Ministry of Education, P.O. Box 270, Hefei, \\ Anhui 230009, China \\ Correspondence should be addressed to Min Xue; xuehfut@163.com
}

Received 26 May 2016; Accepted 1 August 2016

Academic Editor: Yong Deng

Copyright (C) 2016 Min Xue et al. This is an open access article distributed under the Creative Commons Attribution License, which permits unrestricted use, distribution, and reproduction in any medium, provided the original work is properly cited.

\begin{abstract}
Bidimensional dual hesitant fuzzy (BDHF) set is developed to present preferences of a decision maker or an expert, which is more objective than existing fuzzy sets such as Atanassov's intuitionistic fuzzy set, hesitant fuzzy set, and dual hesitant fuzzy set. Then, after investigating some distance measures, we define a new generalized distance measure between two BDHF elements with parameter $\lambda$ for the sake of overcoming some drawbacks in existing distance measures. Covering all possible values of parameter $\lambda$, a new approach is designed to calculate the generalized distance measure between two BDHF elements. In order to address complex multiple attribute decision analysis (MADA) problems, an extension of fuzzy VIKOR method in BDHF context is proposed in this paper. In VIKOR method for MADA problems, weight of each attribute indicates its relative importance. To obtain weights of attributes objectively, a new entropy measure with BDHF information is developed to create weight of each attribute. Finally, an evaluation problem of performance of people's livelihood project in several regions is analyzed by the proposed VIKOR method to demonstrate its applicability and validity.
\end{abstract}

\section{Introduction}

Multiple attribute decision analysis (MADA) is used to deal with the problems of making an optimal choice from alternatives or generating a ranking order of alternatives in terms of attributes $[1,2]$. In order to deal with MADA problems, many decision analysis methods have been proposed, such as outranking method $[3,4]$, TOPSIS (technique for order preference by similarity to ideal solution) [5-7] method, aggregation operator-based methods $[8,9]$, and VIKOR (VIšeKriterijumska Optimizacija I Kompromisno Rešenje) $[10,11]$. Hereinto, due to the characteristics and capabilities of VIKOR method, it has received much attention from academic and practical fields [12, 13]. VIKOR method mainly focuses on ranking and selecting from a set of alternatives and determines compromise solutions for a MADA problem with conflicting attributes $[14,15]$. It represents "closeness to the ideal" in the process of addressing MADA problems. In general, VIKOR method with respect to MADA includes three major steps: (1) the collection of a decision maker's preferences; (2) the calculation of closeness to the ideal; and (3) the determination of attribute weights.

In practical MADA problems, the decision maker may feel difficulty in expressing his/her preferences exactly for the reasons of environmental uncertainty, the limitation of his/her knowledge and experience, and the urgency of time. To address such situations, many expression forms are developed to express information of a decision maker, such as interval-valued number [16], linguistic term set [17], fuzzy set $[18,19]$, hesitant fuzzy (HF) set [20], Atanassov's intuitionistic fuzzy (AIF) set [3, 21], and dual hesitant fuzzy (DHF) set [22]. In some situations, they are not available. Taking a supplier selection problem as an example, there are a supplier denoted by $a_{1}$ and three experts denoted by $e_{1}, e_{2}$, and $e_{3}$. Suppose that two experts (e.g., $e_{1}$ and $e_{2}$ ) consider the preference intensity that supplier $a_{1}$ should be selected and deselected to be 0.7 and 0.1 , respectively. Meanwhile, an expert (e.g., $e_{3}$ ) considers the preference intensity that supplier $a_{1}$ should be selected and deselected is 0.5 and 0.3 , respectively. Therefore, 
the overall preference of the three experts can be denoted by $(\{0.7,0.5\},\{0.1,0.3\})$ which is a DHF assessment. Here, the preference intensity that supplier $a_{1}$ should be selected or deselected means the membership degree or nonmembership degree of experts' preference, respectively. The DHF assessment seems to express the whole preference of the three experts in this supplier selection problem, but it cannot reflect the support intensity of specific membership degrees or that of nonmembership degrees. Here, support intensity means proportion of experts supporting the preference intensity. In the example mentioned above, the support intensity of membership degree 0.7 is different from that of membership degree 0.5 . Such a difference has not been reflected in the DHF assessment. In fact, the support intensity of membership degree 0.7 is $2 / 3 \approx 0.67$, while the support intensity of membership degree 0.5 is $1 / 3 \approx 0.33$, without considering the fact that the differences in support intensity of various assessments could result in different final decisions. Similarly, the support intensity of the other possible nonmembership degrees can be obtained. Thus, it should be noted from the analysis of this example that membership or nonmembership degrees and their support intensity are two aspects of an assessment and should not be neglected in the process of decision making. However, there have been few studies concerning the support intensity of possible membership degrees or that of nonmembership degrees so far. For this reason, in this paper, based on the support intensity and motivated by the idea of DHF set, a new expression form, that is, bidimensional dual hesitant fuzzy (BDHF) set, is developed to present preference information of a decision maker.

In order to address MADA problems with BDHF information, this paper proposes an extended VIKOR method. Firstly, a new generalized distance measure between BDHF sets is proposed to compare alternatives in MADA. This distance measure, as the generalization of existing distance measures, such as AIF distance measure $[23,24]$ and $\mathrm{HF}$ distance measure $[25,26]$, can relax the following two potential conditions of existing HF distance measures: (1) the possible membership degrees in HF element should be arranged in an increasing or decreasing order; and (2) the shorter one in two HF elements can be extended by adding the same value several times until they have the same length. Obviously, it can avoid missing or changing original information provided by the decision maker and may further avoid influence of the two conditions on the final decision result. The details of the distance measure will be demonstrated in Section 3. Moreover, there is parameter $\lambda$ in distance measure impacting the value of distance measure. However, in existing literatures, few studies state how to obtain the value of parameter $\lambda$ scientifically. Thus, to avoid the negative influence of arbitrary or subjective values of parameter $\lambda$ on the value of distance measure between BDHF assessments, an average distance measure covering all the possible types of the generalized distance measures instead of determining an exact value of parameter $\lambda$ is proposed to calculate the distance measure between two BDHF assessments in VIKOR method. Secondly, an objective method of acquiring weight vector (e.g., entropy measure) is extended in BDHF context to objectively determine weight vector of each attribute. Here, corresponding properties of entropy measure are also investigated. Besides, based on the proposed VIKOR method with BDHF information in this paper, an assessment problem of people's livelihood of different regions is demonstrated to verify applicability and validity of the proposed method. Finally, using the information related to the assessment problem, we compare TOPSIS with VIKOR to reflect the advantages of VIKOR method.

The main contributions of this paper include the following: (1) the introduction of bidimensional dual hesitant fuzzy (BDHF) set; (2) the definition of the generalized distance measure between BDHF assessments; (3) the acquisition of attribute weights based on entropy measure with $\mathrm{BDHF}$ information; (4) the comparison between VIKOR and TOPSIS method; and (5) the development of a new VIKOR method with BDHF assessments.

The rest of this paper is organized as follows. Section 2 reviews some basic concepts. Section 3 proposes the concept of BDHF element and new generalized distance measure. Section 4 develops VIKOR method for MADA problems. Section 5 conducts an assessment problem of people's livelihood project to demonstrate the validity and applicability of the proposed method and compares it with TOPSIS method. Finally, Section 6 concludes this paper.

\section{Review of Relevant Concepts}

In this section, we review some concepts related to the proposed MADA method with BDHF information.

2.1. Related Concepts of DHF Set. BDHF set can be regarded as a generalization of DHF set, as mentioned in Introduction. To make the concept of BDHF set clear, the concept of DHF set is introduced below. Here, DHF set can be considered a combination of AIF set and HF set.

Definition 1 (see [22]). Given a universe of discourse $X$, a DHF set on $X$ is defined as

$$
M=\left\{\left\langle x, h_{M}(x), g_{M}(x)\right\rangle \mid x \in X\right\},
$$

where $h_{M}(x)$ and $g_{M}(x)$ symbolize the set of possible membership degrees and the set of nonmembership degrees of $x$ to $M$, respectively, such that $h_{M}(x): X \rightarrow[0,1], g_{M}(x)$ : $X \rightarrow[0,1]$, and $0 \leq \max \left\{h_{M}(x)\right\}+\max \left\{g_{M}(x)\right\} \leq 1$ for all $x \in X$.

Given $h_{M}(x)$ and $g_{M}(x), \quad f_{M}(x)$ = $\bigcup_{\gamma_{M}(x) \in h_{M}(x), \eta_{M}(x) \in g_{M}(x)}\left\{1-\gamma_{M}(x)-\eta_{M}(x)\right\}$ is defined to symbolize possible indeterminacy (uncertain) set of $x$ to $M$, where $\gamma_{M}(x)$ and $\eta_{M}(x)$ represent possible membership and nonmembership degrees of $x$ to $M$. When $x \in X$ is given, $\alpha=(h, g)=\left(\bigcup_{\gamma \in h_{M}(x)}\{\gamma\}, \bigcup_{\eta \in g_{M}(x)}\{\eta\}\right)$ is named as a DHF element, where $\bigcup_{\gamma \in h_{M}(x), \eta \in g_{M}(x)}\{1-\gamma-\eta\}$ denotes possible indeterminacy (uncertain) set of $x$ to $M$.

It is noted that the lengths of different HF sets may be different and thus different DHF sets may also have various lengths. 
2.2. Distance Measure between AIF Information and That between HF Information. When VIKOR method is applied to analyze MADA problems, distances between decision information and ideal solutions are used to compare alternatives. As such, distance measure between BDHF information is needed for the developed method. Meanwhile, as a generalization of DHF set, $\mathrm{BDHF}$ set is closely related to AIF set and HF set. In order to effectively measure the distance among BDHF information, the existing generalized distance measure among AIF information and that among $\mathrm{HF}$ information are defined below.

Definition 2 (see [24]). Let $\alpha_{1}=\left(u_{1}, v_{1}\right)$ and $\alpha_{2}=\left(u_{2}, v_{2}\right)$ be two AIF numbers. Then, a generalized distance measure between $\alpha_{1}$ and $\alpha_{2}$ is defined as

$$
d\left(\alpha_{1}, \alpha_{2}\right)=\left\{\frac{1}{2}\left(\left|u_{1}-u_{2}\right|^{\lambda}+\left|v_{1}-v_{2}\right|^{\lambda}\right)\right\}^{1 / \lambda}
$$

where $\lambda \geq 0$ represents a parameter to indicate different specific distance measures.

Definition 3 (see [26]). Let $h_{1}=\left\{\gamma_{11}, \ldots, \gamma_{1 \delta}\right\}$ and $h_{2}=\left\{\gamma_{21}\right.$, $\left.\ldots, \gamma_{2 \delta}\right\}$ be two HF elements where $l$ symbolizes the length of $h_{1}\left(h_{2}\right)$. Then, a generalized distance measure between $h_{1}$ and $h_{2}$ is defined as

$$
d\left(h_{1}, h_{2}\right)=\left(\frac{1}{l} \sum_{j=1}^{\delta}\left|\gamma_{1 \sigma(j)}-\gamma_{2 \sigma(j)}\right|^{\lambda}\right)^{1 / \lambda}
$$

where $\lambda \geq 0$ is a parameter to signify different specific distance measures and $\gamma_{1 \sigma(j)}$ and $\gamma_{2 \sigma(j)}$ denote the $j$ th largest membership degree in $h_{1}$ and $h_{2}$, respectively. Specifically, $(\sigma(1), \sigma(2), \ldots, \sigma(\delta))$ is a permutation of $(1,2, \ldots, \delta)$ such that $\gamma_{1 \sigma(j)} \geq \gamma_{1 \sigma(j+1)}$ and $\gamma_{2 \sigma(j)} \geq \gamma_{2 \sigma(j+1)}$ for all $j=1,2, \ldots, \delta$.

As mentioned in Section 2.1, it may be the case that the lengths of $h_{1}$ and $h_{2}$ are different in most practices. In order to make the distance measure applicable in such case, $\mathrm{Xu}$ and $\mathrm{Xia}$ [26] and $\mathrm{Xu}$ and Zhang [27] proposed some strategies to equalize the lengths of $h_{1}$ and $h_{2}$. The prerequisite of these ways is to arrange the membership degrees in $h_{1}$ and $h_{2}$ in increasing or decreasing order. Then, the same value will be repeatedly added to the shorter HF element $\left(h_{1}\right.$ or $\left.h_{2}\right)$ until its length is the same as the length of the longer one. There are usually three strategies of adding values:

(1) Optimists expect desirable outcomes and add the maximum value [26].

(2) Pessimists anticipate unfavorable outcomes and may add the minimum value [26].

(3) Neutrals look forward to unbiased outcomes and may add the average value [27].

In real life, even if the risk attitude of a decision maker is clear, the degree to which the decision maker prefers riskseeking or risk-aversion may be also unknown. In other words, a decision maker with risk-seeking to some extent is not certainly sure that the maximum value should be added to the shorter HF element. It is the similar case for pessimists. More importantly, any strategy has no ability to perfectly reflect the original preference of a decision maker, especially when one or more values in HF element appear several times originally.

2.3. Entropy Measure with AIF Information. In MADA, the entropy of decision information is usually employed to determine attribute weights. This idea is also adopted in the developed method. That is, the entropy of BDHF information is measured to create attribute weights. To do this, the entropy of AIF information is presented as necessary foundation.

Definition 4 (see [28]). A real function $I$ : AIF sets $(X) \rightarrow$ $[0,1]$ is called Atanassov's intuitionisitc fuzzy entropy if $I$ satisfies the following axiomatic requirements for all $A, B \in$ AIF sets $(X)$ :

(1) $I(A)=0$ if $A \in 2^{x}$ ( $A$ being nonfuzzy).

(2) $I(A)=1$ if $u_{A}\left(x_{i}\right)=v_{A}\left(x_{i}\right)=0$ for all $x_{i} \in X$.

(3) $I(A) \leq I(B)$ if $A$ is less fuzzy than $B$, that is, $u_{A}(x) \leq$ $u_{B}(x)$ and $v_{A}(x) \geq v_{B}(x)$ for $u_{B}(x) \leq u_{B}(x)$ or $u_{A}(x) \geq u_{B}(x)$ and $v_{A}(x) \leq v_{B}(x)$ for $u_{B}(x) \geq u_{B}(x)$.

(4) $I(A)=I\left(A^{c}\right)$.

Definition 5 (see [29]). Let $A=\left\{\left(x_{i}, u_{A}\left(x_{i}\right), v_{A}\left(x_{i}\right)\right) \mid x_{i} \in X\right\}$ be an AIF set on the universe of discourse $X$. Then, entropy measure $I(A)$ with the AIF set is defined as

$$
\begin{gathered}
I(A)=-\frac{1}{m \ln 2} \sum_{i=1}^{m}\left[u_{A}\left(x_{i}\right) \ln u_{A}\left(x_{i}\right)+v_{A}\left(x_{i}\right)\right. \\
\cdot \ln v_{A}\left(x_{i}\right)-\left(1-\pi_{A}\left(x_{i}\right)\right) \\
\left.\cdot \ln \left(1-\pi_{A}\left(x_{i}\right)\right)-\pi_{A}\left(x_{i}\right) \ln 2\right],
\end{gathered}
$$

where $m$ is the cardinality of the finite universe $X$. This AIF entropy satisfies axiomatic conditions (1)-(4) in Definition 4.

\section{BDHF Set and Distance Measure among BDHF Information}

In this section, BDHF element is defined based on the concept of $\mathrm{DHF}$ element and the distance between BDHF elements is measured in order to develop the VIKOR method with BDHF information.

3.1. Concept of BDHF Set. Reconsidering the supplier selection problem in Introduction, which is a MADA problem, we can find that when three experts give different preferences about selecting and deselecting one supplier, the union of the preferences can naturally form the DHF preference of a decision maker on the assumption that each expert is equivalently important for the decision maker. However, when two or three experts provide the same preference, the resulting DHF preference can be regarded as a transformation from dual intuitionistic fuzzy multiset to DHF set in the abstract [30]. Simply getting the union of the same preference in such transformation will inevitably result in the loss of 
original information. Any strategy in Section 2.2 cannot recover all original information and avoid information loss. To address this issue, we firstly introduce the concept of support intensity given that experts or others used to help a decision maker generate DHF preferences are considered information sources and then define BDHF set based on support intensity.

Definition 6. Suppose a decision maker provides a DHF element $\alpha=(h, g)=\left(\left\{\gamma_{i}(x), i=1, \ldots, m\right\},\left\{\eta_{j}(x), j=1, \ldots\right.\right.$, $n\})$ depending on $T$ information resources. Let the number of information sources supporting membership degree $\gamma_{i}(x)$ and that supporting nonmembership degree $\eta_{j}(x)$ be, respectively, denoted by $T_{\gamma_{i}(x)}$ and $T_{\eta_{j}(x)}$ satisfying $\sum_{i=1}^{m} T_{\gamma_{i}(x)}=T$ and $\sum_{j=1}^{n} T_{\eta_{j}(x)}=T$; then the support intensity of membership degree $\gamma_{i}(x)$ and that of nonmembership degree $\eta_{j}(x)$ are measured by $T_{\gamma_{i}(x)} / T$ and $T_{\eta_{j}(x)} / T$, respectively.

When introducing the concept of support intensity of membership and nonmembership degrees, information implied by dual intuitionistic fuzzy multiset can be effectively covered. That is, when dual intuitionistic fuzzy multiset is transformed into DHF set with support intensity of membership and nonmembership degrees, it can be recovered without information loss. Activated by this idea, DHF set is extended to be BDHF set, as defined below.

Definition 7. Let $X$ be a universe of discourse. A BDHF set on $X$ is defined as

$$
N=\left\{\left\langle x, h_{N}(x), g_{N}(x)\right\rangle \mid x \in X\right\},
$$

where $h_{N}(x)=\left\{\left(\gamma_{i}(x), p_{\gamma_{i}}(x)\right), i=1, \ldots, m\right\}$ and $g_{N}(x)=$ $\left\{\left(\eta_{j}(x), p_{\eta_{j}}(x)\right), j=1, \ldots, n\right\}$ denote possible membership degree of $x$ to $N$ and its support intensity and possible nonmembership degree of $x$ to $N$ and its support intensity, in which $\gamma_{i}(x) \in[0,1], \eta_{j}(x) \in[0,1], \sum_{i=1}^{m} p_{\gamma_{i}}(x)=1$, $\sum_{j=1}^{n} p_{\eta_{j}}(x)=1$, and $0 \leq \max \left\{\gamma_{i}(x)\right\}+\max \left\{\eta_{j}(x)\right\} \leq 1$.

For simplicity, a BDHF element is still denoted by $\alpha=$ $(h, g)$. Here, all original information including membership degree, nonmembership degree, and support intensity with the help of the information resources is effectively characterized in a BDHF element, which cannot be explicitly portrayed in a DHF element.

From Definition 1, it can be inferred that each membership degree in a DHF element can be combined with any nonmembership degree to form Atanassov's intuitionistic fuzzy (AIF) number [22]. Similarly, a BDHF number as the extension of a DHF element can be transformed into several AIF numbers in decision making process such as the computation of distance measure. In this process, besides membership and nonmembership degrees, their support intensity as another important aspect of a BDHF element should be considered so as to complete this decision process. Thus, each AIF number involved in a BDHF element should also have specific support intensity which is a combination of support intensity of each of the membership and nonmembership degrees related to this AIF number. Here, how to obtain the support intensity of an AIF number involved in a $\mathrm{BDHF}$ element is a key problem. In order to tackle this issue, the following assumption is proposed.

Assumption 8. Based on the original information provided by all information resources, a decision maker can provide $\mathrm{BDHF}$ assessments related to some alternatives on several attributes. In this process, it is required for a decision maker that

(1) a BDHF assessment once provided by the decision maker will not be changed no matter whether other BDHF assessments are known or not;

(2) the decision maker specifies membership degrees in a $\mathrm{BDHF}$ assessment without considering nonmembership degrees;

(3) the decision maker specifies nonmembership degrees in a BDHF assessment without considering membership degrees.

Assumption 8 means that any two BDHF assessments are mutually independent and any membership degree and nonmembership degree in a BDHF element are also mutually independent in a decision making process.

Then, under Assumption 8, support intensity of each AIF number involved in a BDHF element can be determined in order to complete all operations in a decision making process including the computation of distance measure as follows.

Definition 9. Let $\alpha=(h, g)=\left(\left\{\left(\gamma_{1}, p_{\gamma_{1}}\right), \ldots,\left(\gamma_{\delta(h)}, p_{\gamma_{\delta(h)}}\right)\right\}\right.$, $\left.\left\{\left(\eta_{1}, p_{\eta_{1}}\right), \ldots,\left(\eta_{\delta(g)}, p_{\eta_{\delta(g)}}\right)\right\}\right)$ be a BDHF element where $\delta(h)$ and $\delta(g)$ symbolize the numbers of possible membership and nonmembership degrees, respectively. The combination of each AIF number and support intensity involved in a BDHF element can be defined as

$$
\begin{aligned}
\alpha & =(h, g)=\bigcup_{\left(\gamma_{i}, p_{\gamma_{i}}\right) \in h,\left(\eta_{j}, p_{\eta_{j}}\right) \in g}\left\{\left(\gamma_{i}, p_{\gamma_{i}}\right),\left(\eta_{j}, p_{\eta_{j}}\right)\right\} \\
& =\bigcup_{\left(\gamma_{i}, p_{\gamma_{i}}\right) \in h,\left(\eta_{j}, p_{\eta_{j}}\right) \in g}\left\{\left(\gamma_{i}, \eta_{j}\right),\left(p_{\gamma_{i}} \cdot p_{\eta_{j}}\right)\right\} .
\end{aligned}
$$

Here, under Assumption 8, because of the independence between membership degree and nonmembership degree in any AIF number, it can be inferred that support intensity of an AIF number involved in a BDHF element is the product of that of corresponding membership and nonmembership degrees. Thus, the number of the AIF numbers involved in a $\mathrm{BDHF}$ element is $\delta(h) \times \delta(g)$. For example, given a $\mathrm{BDHF}$ element denoted by $\alpha=(\{(0.2,0.6),(0.3,0.4)\},\{(0.5,1)\})$, then the combination of each AIF number involved in the BDHF element can be obtained as $\alpha=(\{(0.2,0.6),(0.3,0.4)\}$, $\{(0.5,1)\})=(\{(0.2,0.5),(0.6 \cdot 1)\},\{(0.3,0.5),(0.4 \cdot 1)\})$.

In addition, it can be obtained from Definition 1 that possible indeterminacy (uncertain) set of $x$ to DHF set $M$ is equal to $\bigcup_{\gamma \in h_{M}(x), \eta \in g_{M}(x)}\{1-\gamma-\eta\}$ which means the union where one subtracts the sum of any possible membership and nonmembership degrees. However, in a BDHF element, not only membership and nonmembership degrees but also 
their corresponding support intensity should be considered. Thus, based on Assumption 8 and Definition 9, we can infer that support intensity of possible indeterminacy (uncertain) degree of $x$ to BDHF set $N$ equals the product of support intensity of corresponding membership and nonmembership degrees, which is demonstrated in the following:

$$
f_{N}(x)=\bigcup_{\left(\gamma_{i}, p_{\gamma_{i}}\right) \in h_{N}(x),\left(\eta_{j}, p_{\eta_{j}}\right) \in g_{N}(x)}\left(1-\gamma_{i}-\eta_{j}, p_{\gamma_{i}} p_{\eta_{j}}\right),
$$

where $f_{N}(x)$ symbolizes possible indeterminacy (uncertain) set of $x$ to $N$ and $\left(\gamma_{i}, p_{\gamma_{i}}\right)$ and $\left(\eta_{j}, p_{\eta_{j}}\right)$ represent possible membership and nonmembership degrees and their corresponding support intensity of $x$ to $N$.

Then, the basic operations of BDHF elements can be defined in the following.

Definition 10. Let $X$ be a fixed set and $\alpha_{1}=\left(h_{1}, g_{1}\right)$ and $\alpha_{2}=$ $\left(h_{2}, g_{2}\right)$ be two BDHF elements; the following operations are valid:

(1) $\alpha_{1} \oplus \alpha_{2}=\left(\bigcup_{\left(\gamma_{1}, p_{\gamma_{1}}\right) \in h_{1},\left(\gamma_{2}, p_{\gamma_{2}}\right) \in h_{2}}\left\{\left(\gamma_{1}+\gamma_{2}-\gamma_{1} \gamma_{2}, p_{\gamma_{1}} \cdot p_{\gamma_{2}}\right)\right\}\right.$, $\left.\bigcup_{\left(\eta_{1}, p_{\eta_{1}}\right) \in g_{1},\left(\eta_{2}, p_{\eta_{2}}\right) \in g_{2}}\left\{\left(\eta_{1} \eta_{2}, p_{\eta_{1}} \cdot p_{\eta_{2}}\right)\right\}\right)$.

(2) $\alpha_{1} \otimes \alpha_{2}=\left(\bigcup_{\left(\gamma_{1}, p_{\gamma_{1}}\right) \in h_{1},\left(\gamma_{2}, p_{\gamma_{2}}\right) \in h_{2}}\left\{\left(\gamma_{1} \gamma_{2}, p_{\gamma_{1}} \cdot p_{\gamma_{2}}\right)\right\}\right.$, $\left.\bigcup_{\left(\eta_{1}, p_{\eta_{1}}\right) \in g_{1},\left(\eta_{2}, p_{\eta_{2}}\right) \in g_{2}}\left\{\left(\eta_{1}+\eta_{2}-\eta_{1} \eta_{2}, p_{\eta_{1}} \cdot p_{\eta_{2}}\right)\right\}\right)$.

(3) $\lambda \alpha_{1}=\left(\bigcup_{\left(\gamma_{1}, p_{\gamma_{1}}\right) \in h_{1}}\left\{\left(1-\left(1-\gamma_{1}\right)^{\lambda}, p_{\gamma_{1}}^{\lambda}\right)\right\}, \bigcup_{\left(\eta_{1}, p_{\eta_{1}}\right) \in g_{1}}\left\{\left(\eta_{1}^{\lambda}\right.\right.\right.$, $\left.\left.\left.p_{\eta_{1}}^{\lambda}\right)\right\}\right), \lambda>0$.

(4) $\alpha_{1}^{\lambda}=\left(\bigcup_{\left(\gamma_{1}, p_{\gamma_{1}}\right) \in h_{1}}\left\{\left(\gamma_{1}^{\lambda}, p_{\gamma_{1}}^{\lambda}\right)\right\}, \bigcup_{\left(\eta_{1}, p_{\eta_{1}}\right) \in g_{1}}\left\{\left(1-\left(1-\eta_{1}\right)^{\lambda}\right.\right.\right.$, $\left.\left.\left.p_{\eta_{1}}^{\lambda}\right)\right\}\right), \lambda>0$.

(5) $\alpha_{1}^{c}=\left(\bigcup_{\left(\eta_{1}, p_{\eta_{1}}\right) \in g_{1}}\left\{\left(\eta_{1}, p_{\eta_{1}}\right)\right\}, \bigcup_{\left(\gamma_{1}, p_{\gamma_{1}}\right) \in h_{1}}\left\{\left(\gamma_{1}, p_{\gamma_{1}}\right)\right\}\right)$.

Here, $\alpha_{1}^{c}$ represents the complement of the BDHF element $\alpha_{1}$.

3.2. Distance Measure between BDHF Elements. As mentioned in Introduction, the computation of distance measure between BDHF elements is a key step in the proposed VIKOR method. From Definition 11, it can be obtained that, in the process of measuring distance between two BDHF elements, any AIF number involved in one BDHF element and that in another BDHF element should be covered. That is, distance measure between two AIF numbers contributes to that between two BDHF elements. Thus, in order to define the distance measure between two BDHF elements, we should first design distance measure between two AIF numbers involved in two BDHF elements below.

Definition 11. Let $\beta_{1}=\left(\gamma_{1}, \eta_{1}\right)$ and $\beta_{2}=\left(\gamma_{2}, \eta_{2}\right)$ be any two AIF numbers from two BDHF elements, respectively. Then, the distance measure between $\beta_{1}$ and $\beta_{2}$ can be defined as follows:

$$
d\left(\beta_{1}, \beta_{2}\right)=\left(0.5 \cdot\left(\left|\gamma_{1}-\gamma_{2}\right|^{\lambda}+\left|\eta_{1}-\eta_{2}\right|^{\lambda}\right)\right)^{1 / \lambda}
$$

where $\lambda \geq 1$ is a parameter to signify different distance measures.

From Definition 9, besides distance measure between AIF numbers, the support intensity of each AIF number should be considered to calculate distance measure between BDHF elements. Then, under Assumption 8, because of the independence among any two BDHF elements, it can be obtained from Definition 9 that the support intensity of distance measure between two AIF numbers involved in two BDHF elements equals the product of the support intensity of these two AIF numbers, which is defined in the following.

Definition 12. Let $\beta_{11}=\left\{\left(\gamma_{11}, \eta_{11}\right),\left(p_{\gamma_{11}} \cdot p_{\eta_{11}}\right)\right\}$ and $\beta_{21}=$ $\left\{\left(\gamma_{21}, \eta_{21}\right),\left(p_{\gamma_{21}} \cdot p_{\eta_{21}}\right)\right\}$ be two combinations of two AIF numbers and their support intensity involved in two BDHF elements, respectively. Then, the support intensity of distance measure between $\beta_{11}$ and $\beta_{21}$ can be defined as follows:

$$
p\left(d\left(\beta_{11}, \beta_{21}\right)\right)=p_{\gamma_{11}} p_{\eta_{11}} \cdot p_{\gamma_{21}} p_{\eta_{21}},
$$

where $p_{\gamma_{11}}$ and $p_{\eta_{11}}$ denote support intensity of the 1st possible membership degree and the 1st possible nonmembership degree in $\alpha_{1}$, respectively.

Because the distance measure between BDHF elements consists of all combinations of AIF numbers with their support intensity, then, combining Definition 11 with Definition 12 , the distance measure between two BDHF elements can be defined below.

Definition 13. Let $\alpha_{1}=\left(h_{1}, g_{1}\right)=\left(\left\{\left(\gamma_{11}, p_{\gamma_{11}}\right), \ldots,\left(\gamma_{1 \delta\left(h_{1}\right)}\right)\right.\right.$, $\left.\left.\left.p_{\gamma_{1 \delta\left(h_{1}\right)}}\right)\right\},\left\{\left(\eta_{11}, p_{\eta_{11}}\right), \ldots,\left(\eta_{1 \delta\left(g_{1}\right)}, p_{\eta_{1 \delta\left(g_{1}\right)}}\right)\right\}\right)$ and $\alpha_{2}=\left(h_{2}\right.$, $\left.g_{2}\right)=\left(\left\{\left(\gamma_{21}, p_{\gamma_{21}}\right), \ldots,\left(\gamma_{2 \delta\left(h_{2}\right)}, p_{\gamma_{2 \delta\left(h_{2}\right)}}\right)\right\},\left\{\left(\eta_{21}, p_{\eta_{21}}\right), \ldots\right.\right.$,

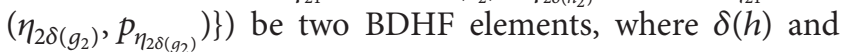
$\delta(g)$ symbolize the numbers of possible membership and nonmembership degrees, respectively. Then, the generalized distance measure between $\alpha_{1}$ and $\alpha_{2}$ can be defined as follows:

$$
\begin{aligned}
d\left(\alpha_{1}, \alpha_{2}\right) & =\sum_{i=1}^{\delta\left(h_{1}\right)} \sum_{j=1}^{\delta\left(g_{1}\right)} \sum_{p=1}^{\delta\left(h_{2}\right)} \sum_{q=1}^{\delta\left(g_{2}\right)} p_{\gamma_{1 i}} p_{\gamma_{2 p}} p_{\eta_{1 j}} p_{\eta_{2 q}} \cdot d\left(\beta_{i j}, \beta_{p q}\right) \\
& =\sum_{i=1}^{\delta\left(h_{1}\right)} \sum_{j=1}^{\delta\left(g_{1}\right)} \sum_{p=1}^{\delta\left(h_{2}\right)} \sum_{q=1}^{\delta\left(g_{2}\right)} p_{\gamma_{1 i}} p_{\gamma_{2 p}} p_{\eta_{1 j}} p_{\eta_{2 q}}\left(0.5 \cdot\left(\left|\gamma_{1 i}-\gamma_{2 p}\right|^{\lambda}+\left|\eta_{1 j}-\eta_{2 q}\right|^{\lambda}\right)\right)^{1 / \lambda},
\end{aligned}
$$

where $p_{\gamma_{1 i}}$ and $p_{\eta_{1 j}}\left(i=1, \ldots, \delta\left(h_{1}\right) ; j=1, \ldots, \delta\left(g_{1}\right)\right)$ denote support intensity of the $i$ th possible membership degree and the $j$ th possible nonmembership degree in $\alpha_{1}$, respectively. Similarly, $p_{\gamma_{2 p}}$ and $p_{\eta_{2 q}}\left(p=1, \ldots, \delta\left(h_{2}\right) ; q=1, \ldots, \delta\left(g_{2}\right)\right)$ 
denote support intensity of the $p$ th possible membership degree and the $q$ th possible nonmembership degree in $\alpha_{2}$, respectively. In addition, $d\left(\beta_{i j}, \beta_{p q}\right)$ can be obtained from Definition 11.

As analyzed in Section 2.2, the distance measure between HF elements in Definition 3 is based on several conditions which will constrain the application of this distance measure. From Definition 13, it is obvious that these conditions are relaxed.

Then, in order to demonstrate the process of calculating the distance measure in Definition 13 clearly, Example 14 is showed in the following.

Example 14. Let $\alpha_{1}=(\{(0.2,0.6),(0.3,0.4)\},\{(0.5,0.3),(0.6$, $0.4),(0.7,0.3)\})$ and $\alpha_{2}=(\{(0.7,1)\},\{(0.1,0.2),(0.2,0.8)\})$ be two BDHF elements. Then, the generalized distance measure between $\alpha_{1}$ and $\alpha_{2}$ is calculated by Definition 13 as follows:

$$
\begin{aligned}
d\left(\alpha_{1}, \alpha_{2}\right)= & 0.6 \times 1 \times 0.3 \times 0.2 \\
& \times\left(\frac{1}{2}\left[|0.2-0.7|^{\lambda}+|0.5-0.1|^{\lambda}\right]\right)^{1 / \lambda} \\
& +0.6 \times 1 \times 0.3 \times 0.8 \\
& \times\left(\frac{1}{2}\left[|0.2-0.7|^{\lambda}+|0.5-0.2|^{\lambda}\right]\right)^{1 / \lambda} \\
& +0.6 \times 1 \times 0.4 \times 0.2 \\
& \times\left(\frac{1}{2}\left[|0.2-0.7|^{\lambda}+|0.6-0.1|^{\lambda}\right]\right)^{1 / \lambda} \\
& +0.6 \times 1 \times 0.4 \times 0.8 \\
& \times\left(\frac{1}{2}\left[|0.2-0.7|^{\lambda}+|0.6-0.2|^{\lambda}\right]\right)^{1 / \lambda} \\
& +0.6 \times 1 \times 0.3 \times 0.2 \\
& \times\left(\frac{1}{2}\left[|0.2-0.7|^{\lambda}+|0.7-0.1|^{\lambda}\right]\right)^{1 / \lambda} \\
& +0.6 \times 1 \times 0.3 \times 0.8
\end{aligned}
$$

$$
\begin{aligned}
& \times\left(\frac{1}{2}\left[|0.2-0.7|^{\lambda}+|0.7-0.2|^{\lambda}\right]\right)^{1 / \lambda} \\
& +0.4 \times 1 \times 0.3 \times 0.2 \\
& \times\left(\frac{1}{2}\left[|0.3-0.7|^{\lambda}+|0.5-0.1|^{\lambda}\right]\right)^{1 / \lambda} \\
& +0.4 \times 1 \times 0.3 \times 0.8 \\
& \times\left(\frac{1}{2}\left[|0.3-0.7|^{\lambda}+|0.5-0.2|^{\lambda}\right]\right)^{1 / \lambda} \\
& +0.4 \times 1 \times 0.4 \times 0.2 \\
& \times\left(\frac{1}{2}\left[|0.3-0.7|^{\lambda}+|0.6-0.1|^{\lambda}\right]\right)^{1 / \lambda} \\
& +0.4 \times 1 \times 0.4 \times 0.8 \\
& \times\left(\frac{1}{2}\left[|0.3-0.7|^{\lambda}+|0.6-0.2|^{\lambda}\right]\right)^{1 / \lambda} \\
& +0.4 \times 1 \times 0.3 \times 0.2 \\
& \times\left(\frac{1}{2}\left[|0.3-0.7|^{\lambda}+|0.7-0.1|^{\lambda}\right]\right)^{1 / \lambda} \\
& +0.4 \times 1 \times 0.3 \times 0.8 \\
& \times\left(\frac{1}{2}\left[|0.3-0.7|^{\lambda}+|0.7-0.2|^{\lambda}\right]\right)^{1 / \lambda} .
\end{aligned}
$$

Obviously, it can be seen from Example 14 that the value of distance measure between $\alpha_{1}$ and $\alpha_{2}$ depends on the value of parameter $\lambda$. The final result will be demonstrated in Example 24.

Three special cases of the generalized distance measure in Definition 13 are presented in the following definitions.

Definition 15. When $\lambda=1$, the generalized distance measure in Definition 13 reduces to the Hamming distance measure:

$$
d\left(\alpha_{1}, \alpha_{2}\right)=\sum_{i=1}^{\delta\left(h_{1}\right)} \sum_{j=1}^{\delta\left(g_{1}\right)} \sum_{p=1}^{\delta\left(h_{2}\right)} \sum_{q=1}^{\delta\left(g_{2}\right)} p_{\gamma_{1 i}} p_{\gamma_{2 p}} p_{\eta_{1 j}} p_{\eta_{2 q}}\left(0.5 \cdot\left(\left|\gamma_{1 i}-\gamma_{2 p}\right|+\left|\eta_{1 j}-\eta_{2 q}\right|\right)\right) .
$$

Definition 16. When $\lambda=2$, the generalized distance measure in Definition 13 reduces to the Euclidean distance measure:

$$
d\left(\alpha_{1}, \alpha_{2}\right)=\sum_{i=1}^{\delta\left(h_{1}\right)} \sum_{j=1}^{\delta\left(g_{1}\right)} \sum_{p=1}^{\delta\left(h_{2}\right)} \sum_{q=1}^{\delta\left(g_{2}\right)} p_{\gamma_{1 i}} p_{\gamma_{2 p}} p_{\eta_{1 j}} p_{\eta_{2 q}}\left(0.5 \cdot\left(\left|\gamma_{1 i}-\gamma_{2 p}\right|^{2}+\left|\eta_{1 j}-\eta_{2 q}\right|^{2}\right)\right)^{1 / 2} .
$$


Definition 17. When $\lambda=+\infty$, the generalized distance measure in Definition 13 reduces to the Chebyshev distance measure:

$$
\begin{aligned}
& d\left(\alpha_{1}, \alpha_{2}\right) \\
& =\sum_{i=1}^{\delta\left(h_{1}\right)} \sum_{j=1}^{\delta\left(g_{1}\right)} \sum_{p=1}^{\delta\left(h_{2}\right)} \sum_{q=1}^{\delta\left(g_{2}\right)} p_{\gamma_{1 i}} p_{\gamma_{2 p}} p_{\eta_{1 j}} p_{\eta_{2 q}}\left(\max \left[\left|\gamma_{1 i}-\gamma_{2 j}\right|,\left|\eta_{1 i}-\eta_{2 j}\right|\right]\right) .
\end{aligned}
$$

The generalized distance measure in Definition 13 has some properties including boundedness, commutativity, and conditional reflexivity, which are concluded in the following theorem.

Theorem 18. Given two BDHF elements, $\alpha_{1}=\left(h_{1}\right.$, $\left.g_{1}\right)=\left(\left\{\left(\gamma_{11}, p_{\gamma_{11}}\right), \ldots,\left(\gamma_{1 \delta\left(h_{1}\right)}, p_{\gamma_{1 \delta\left(h_{1}\right)}}\right)\right\},\left\{\left(\eta_{11}, p_{\eta_{11}}\right), \ldots\right.\right.$, $\left.\left.\left(\eta_{1 \delta\left(g_{1}\right)}, p_{\eta_{1 \delta\left(g_{1}\right)}}\right)\right\}\right)$ and $\alpha_{2}=\left(h_{2}, g_{2}\right)=\left(\left\{\left(\gamma_{21}, p_{\gamma_{21}}\right), \ldots,\left(\gamma_{2 \delta\left(h_{2}\right)}\right.\right.\right.$, $\left.\left.\left.p_{\gamma_{2 \delta\left(h_{2}\right)}}\right)\right\},\left\{\left(\eta_{21}, p_{\eta_{21}}\right), \ldots,\left(\eta_{2 \delta\left(g_{2}\right)}, p_{\eta_{2 \delta\left(g_{2}\right)}}\right)\right\}\right)$, the generalized distance measure in Definition 13 satisfies the following properties:

(1) Boundedness is $0 \leq d\left(\alpha_{1}, \alpha_{2}\right) \leq 1$.

(2) Commutativity is $d\left(\alpha_{1}, \alpha_{2}\right)=d\left(\alpha_{2}, \alpha_{1}\right)$.

(3) Conditional reflexivity is $d\left(\alpha_{1}, \alpha_{2}\right)=0$ if $\delta\left(h_{1}\right)=$ $\delta\left(h_{2}\right)=\delta\left(g_{1}\right)=\delta\left(g_{2}\right)=1, \gamma_{11}=\gamma_{21}, \eta_{11}=\eta_{21}$, and $p_{\gamma_{11}}=p_{\gamma_{21}}=p_{\eta_{11}}=p_{\eta_{21}}=1$.

Theorem 18 is proven in Section A.1 of Appendix A.

As mentioned in Definition 13, parameter $\lambda$ is limited to $[1,+\infty)$. Determining the precise value of $\lambda$ from the interval $[1,+\infty)$ may be difficult, especially when various kinds of information are involved in the determination process. However, most existing studies arbitrarily or subjectively assign several special values to parameter $\lambda$ without reasonable explanation, which may not always accord with practical situations. More importantly, it may lead to an unrealistic distance between two elements without appropriate parameter $\lambda$. To avoid the negative influence of arbitrary or subjective $\lambda$ on distance between two elements, we use average value of the generalized distance measure between two BDHF elements to calculate the final distance measure with full coverage of the values of possible $\lambda$. Hereinto, the average value of the generalized distance measure could be considered a most likely result covering all possible types of distance measures.

Definition 19 (see [31]). If a function $g$ is integrable on interval $[a, b]$, then the average value of $f$ denoted by $\bar{g}$ on $[a, b]$ can be defined as follows:

$$
\bar{g}=\frac{1}{b-a} \int_{a}^{b} f(x) d x
$$

Based on Definitions 13 and 19, the average value of the generalized distance measure is designed to calculate the final distance measure, which is presented as follows.

Definition 20. Let $\quad d\left(\alpha_{1}, \alpha_{2}\right)=$ $\sum_{i=1}^{\delta\left(h_{1}\right)} \sum_{j=1}^{\delta\left(g_{1}\right)} \sum_{p=1}^{\delta\left(h_{2}\right)} \sum_{q=1}^{\delta\left(g_{2}\right)} p_{\gamma_{1 i}} p_{\gamma_{2 p}} p_{\eta_{1 j}} p_{\eta_{2 q}}\left(0.5 \cdot\left(\left|\gamma_{1 i}-\gamma_{2 p}\right|^{\lambda}+\right.\right.$ $\left.\left.\left|\eta_{1 j}-\eta_{2 q}\right|^{\lambda}\right)\right)^{1 / \lambda}$ be a generalized distance measure between two BDHF elements. Then the average value of the generalized distance measure is defined as

$$
\overline{d\left(\alpha_{1}, \alpha_{2}\right)}=\lim _{\lambda_{0} \rightarrow+\infty}\left(\int_{1}^{\lambda_{0}} \sum_{i=1}^{\delta\left(h_{1}\right)} \sum_{j=1}^{\delta\left(g_{1}\right)} \sum_{p=1}^{\delta\left(h_{2}\right)} \sum_{q=1}^{\delta\left(g_{2}\right)} \frac{p_{\gamma_{1 i}} p_{\gamma_{2 p}} p_{\eta_{1 j}} p_{\eta_{2 q}}\left(0.5 \cdot\left(\left|\gamma_{1 i}-\gamma_{2 p}\right|^{\lambda}+\left|\eta_{1 j}-\eta_{2 q}\right|^{\lambda}\right)\right)^{1 / \lambda} d \lambda}{\left(\lambda_{0}-1\right)}\right)
$$

In this equation, it is obvious that $\lim _{\lambda_{0} \rightarrow+\infty}\left(\lambda_{0}-1\right)$ is divergent. Thus, L'Hopital's Rule could be applied to calculate the value of $\overline{d\left(\alpha_{1}, \alpha_{2}\right)}$ as long as the following two conditions are satisfied:

(1) The numerator of $\overline{d\left(\alpha_{1}, \alpha_{2}\right)}$ diverges.

(2) $\lim _{\lambda \rightarrow+\infty}\left[\left(\int_{1}^{\lambda} \sum_{i=1}^{\delta\left(h_{1}\right)} \sum_{j=1}^{\delta\left(g_{1}\right)} \sum_{p=1}^{\delta\left(h_{2}\right)} \sum_{q=1}^{\delta\left(g_{2}\right)} p_{\gamma_{1 i}} p_{\gamma_{2 p}} p_{\eta_{1 j}}\right.\right.$. $\left.\left.p_{\eta_{2 q}}\left(0.5 \cdot\left(\left|\gamma_{1 i}-\gamma_{2 p}\right|^{\lambda}+\left|\eta_{1 j}-\eta_{2 q}\right|^{\lambda}\right)\right)^{1 / \lambda} d \lambda\right) d \lambda /(\lambda-1) d \lambda\right]$ exists.

To achieve the mentioned work simply, we firstly design a new function denoted by $f(\lambda)=\left(0.5\left(a^{\lambda}+b^{\lambda}\right)\right)^{1 / \lambda}(a$, $b \in[0,1])$ representing a major part of $\overline{d\left(\alpha_{1}, \alpha_{2}\right)}$. That is, if the average value of $f(\lambda)$ denoted by $\overline{f(\lambda)}=$ $\lim _{\lambda_{0} \rightarrow+\infty}\left(\int_{1}^{\lambda_{0}} f(\lambda) d \lambda /\left(\lambda_{0}-1\right)\right)$ can be acquired, then the value of $\overline{d\left(\alpha_{1}, \alpha_{2}\right)}$ can be also calculated. Then, we investigate whether $\lim _{\lambda_{0} \rightarrow+\infty} \int_{1}^{\lambda_{0}} f(\lambda) d \lambda$ diverges and $\lim _{\lambda_{0} \rightarrow+\infty}\left(\left(\int_{1}^{\lambda} f(\lambda) d \lambda\right) d \lambda /(\lambda-1) d \lambda\right)$ exists or not. Firstly, monotonicity of function $f(\lambda)$ with respect to $\lambda$ is demonstrated in the following.

Lemma 21. Suppose that $f(\lambda)=\left(0.5\left(a^{\lambda}+b^{\lambda}\right)\right)^{1 / \lambda}$ is a function with parameter $\lambda$ where $a, b \in[0,1]$ and $\lambda \geq 1$. Then, $f(\lambda)$ is monotonous increasing with respect to $\lambda$ and its upper bound is $\max (a, b)$, that is,

$$
\max (a, b) \geq\left(0.5\left(a^{\lambda}+b^{\lambda}\right)\right)^{1 / \lambda}
$$

Lemma 21 is proven in Section A.2 of Appendix A. It can be inferred from Lemma 21 that when $\lambda$ approaches $+\propto, f(\lambda)$ will approximate to $\max (a, b)$. It means that $\lim _{\lambda_{0} \rightarrow+\infty} \int_{1}^{\lambda_{0}} f(\lambda) d \lambda$ is divergent. In addition, it can be inferred from Lemma 21 that $\lim _{\lambda \rightarrow+\infty}\left(\left(\int_{1}^{\lambda} f(\lambda) d \lambda\right) d \lambda /(\lambda-\right.$ 1)d $d \lambda=\lim _{\lambda \rightarrow+\infty} f(\lambda) / 1=\max (a, b)$. Thus, L'Hopital's Rule 
for forms of type $\infty / \infty$ in Lemma 22 could be applied to calculate the value of $\overline{f(\lambda)}$ in Theorem 23 .

Lemma 22 (see [31]). Suppose that $\lim _{x \rightarrow u}|\varphi(x)|=$ $\lim _{x \rightarrow u}|\phi(x)|=\infty$. If $\lim _{x \rightarrow u}\left[\varphi^{\prime}(x) / \phi^{\prime}(x)\right]$ exists in either the finite or infinite sense, then

$$
\lim _{x \rightarrow u} \frac{\varphi(x)}{\phi(x)}=\lim _{x \rightarrow u} \frac{\varphi^{\prime}(x)}{\phi^{\prime}(x)} .
$$

Here, $u$ may stand for any of the symbols $a, a^{-}, a^{+},-\infty$, or $+\infty$.

Theorem 23. Suppose that $f(\lambda)=\left(0.5\left(a^{\lambda}+b^{\lambda}\right)\right)^{1 / \lambda}$, where $a, b \in[0,1]$ and $\lambda \geq 1$. Then, the average value of $f(\lambda)$ denoted by $\overline{f(\lambda)}=\lim _{\lambda_{0} \rightarrow+\infty}\left(\int_{1}^{\lambda_{0}} f(\lambda) d \lambda /\left(\lambda_{0}-1\right)\right)$ equals $\max (a, b)$.

Theorem 23 is proven in Section A.3 of Appendix A with the help of Lemma 21. It indicates from Theorem 23 that $\max (a, b)$ will represent the value of $\overline{f(\lambda)}$ in the process of calculating a distance measure between two elements. Then, it can be obtained that the value of $\overline{d\left(\alpha_{1}, \alpha_{2}\right)}$ equals $\max \left(\mid \gamma_{1 i}-\right.$ $\left.\gamma_{2 p}|,| \eta_{1 j}-\eta_{2 q} \mid\right)$. Clearly, Theorem 23 can simplify computing process of distance measures and avoid the difficulty of determining appropriate parameter $\lambda$. Based on Theorem 23, Example 14 can be further calculated in Example 24.

Example 24. Let $\alpha_{1}=(\{(0.2,0.6),(0.3,0.4)\},\{(0.5,0.3),(0.6$, $0.4),(0.7,0.3)\})$ and $\alpha_{2}=(\{(0.7,1)\},\{(0.1,0.2),(0.2,0.8)\})$ be two BDHF elements. Then, the distance measure between $\alpha_{1}$ and $\alpha_{2}$ is calculated after Example 14 as follows:

$$
\begin{aligned}
d\left(\alpha_{1}, \alpha_{2}\right)= & 0.6 \times 1 \times 0.3 \times 0.2 \times 0.5+0.6 \times 1 \times 0.3 \\
& \times 0.8 \times 0.5+0.6 \times 1 \times 0.4 \times 0.2 \times 0.5 \\
& +0.6 \times 1 \times 0.4 \times 0.8 \times 0.5+0.6 \times 1 \\
& \times 0.3 \times 0.2 \times 0.6+0.6 \times 1 \times 0.3 \times 0.8 \\
& \times 0.5+0.4 \times 1 \times 0.3 \times 0.2 \times 0.4+0.4 \\
& \times 1 \times 0.3 \times 0.8 \times 0.4+0.4 \times 1 \times 0.4 \\
& \times 0.2 \times 0.5+0.4 \times 1 \times 0.4 \times 0.8 \times 0.4 \\
& +0.4 \times 1 \times 0.3 \times 0.2 \times 0.6+0.4 \times 1 \\
& \times 0.3 \times 0.8 \times 0.5=0.4812 .
\end{aligned}
$$

The key characteristic of the distance measure proposed in this paper is to combine membership or nonmembership degrees with their support intensity. Now, if we do not consider support intensity of membership or nonmembership degrees, could different distance measures between BDHF elements be obtained? It can be calculated in Example 24 that without support intensity $d\left(\alpha_{1}, \alpha_{2}\right)=(0.5+0.5+0.5+0.5+$ $0.6+0.5+0.4+0.4+0.5+0.4+0.6+0.5) / 12=0.4917$. By contrast, considering support intensity we can obtain that $d\left(\alpha_{1}, \alpha_{2}\right)=0.4812$ as mentioned above. Obviously, the results from these two ways are different. Therefore, whether the decision maker considers support intensity of membership or nonmembership degrees may lead to different distance measures. Moreover, in decision making, it is the most important thing for the decision maker to try their best to consider all original information. That is the main reason why support intensity is rational and necessary for the decision making.

After obtaining the generalized distance measure between two BDHF elements, the generalized distance measure between two BDHF sets could also be defined in the following.

Definition 25. Let $A_{1}=\left(h_{1 j}, g_{1 j}\right)(j=1, \ldots, n), A_{2}=$ $\left(h_{2 j}, g_{2 j}\right)(j=1, \ldots, n)$ and $w=\left(w_{1}, \ldots, w_{n}\right)^{T}$ be two BDHF sets and weights for each BDHF element, respectively. Then, the generalized distance between $A_{1}$ and $A_{2}$ is measured by

$$
d\left(A_{1}, A_{2}\right)=\sum_{j=1}^{n} w_{j} \cdot d\left(A_{1 j}, A_{2 j}\right),
$$

where $d\left(A_{1 j}, A_{2 j}\right)$ is calculated by Definition 13 .

Similar to the generalized distance measure in Definition 13, the generalized distance measure in Definition 25 has the same properties as follows.

Theorem 26. Suppose that $A_{1}=\left(h_{1 j}, g_{1 j}\right)=\left(\left\{\left(\gamma_{11}^{(j)}, p_{\gamma_{11}^{(j)}}\right)\right.\right.$, $\left.\left.\ldots,\left(\gamma_{1 \delta\left(h_{1 j}\right)}^{(j)}, p_{\gamma_{1 \delta\left(h_{1 j}\right)}^{(j)}}\right)\right\},\left\{\left(\eta_{11}^{(j)}, p_{\eta_{11}^{(j)}}\right), \ldots,\left(\eta_{1 \delta\left(g_{1 j}\right)}^{(j)}, p_{\eta_{1 \delta\left(g_{1 j}\right)}^{(j)}}\right)\right\}\right)(j=$ $1, \ldots, n), A_{2}=\left(h_{2 j}, g_{2 j}\right)=\left(\left\{\left(\gamma_{21}^{(j)}, p_{\gamma_{21}^{(j)}}\right), \ldots,\left(\gamma_{2 \delta\left(h_{2 j}\right)}^{(j)}\right)\right.\right.$ $\left.\left.\left.p_{\gamma_{2 \delta\left(h_{2 j}\right)}^{(j)}}\right)\right\},\left\{\left(\eta_{21}^{(j)}, p_{\eta_{21}^{(j)}}\right), \ldots,\left(\eta_{2 \delta\left(g_{2 j}\right)}^{(j)}, p_{\eta_{2 \delta\left(g_{2 j}\right)}^{(j)}}\right)\right\}\right)(j=1, \ldots, n)$ and $w=\left(w_{1}, \ldots, w_{n}\right)^{T}$ are two BDHF sets and weights for each $B D H F$ element, respectively. The generalized distances measured by Definition 25 satisfy the following:

(1) Boundedness is $0 \leq d\left(A_{1}, A_{2}\right) \leq 1$.

(2) Commutativity is $d\left(A_{1}, A_{2}\right)=d\left(A_{2}, A_{1}\right)$.

(3) Conditional reflexivity is $d\left(A_{1}, A_{2}\right)=0$ if $\gamma_{1 \delta\left(h_{1 j}\right)}^{(j)}=$ $\gamma_{2 \delta\left(h_{2 j}\right)}^{(j)}, \eta_{1 \delta\left(g_{1 j}\right)}^{(j)}=\eta_{2 \delta\left(g_{2 j}\right)}^{(j)}, \delta\left(h_{1 j}\right)=\delta\left(h_{2 j}\right)=\delta\left(g_{1 j}\right)=$ $\delta\left(g_{2 j}\right)=1$ and $p_{\gamma_{1 \delta\left(h_{1 j}\right)}^{(j)}}=p_{\gamma_{2 \delta\left(h_{2 j}\right)}^{(j)}}=p_{\eta_{1 \delta\left(g_{1 j}\right)}^{(j)}}=p_{\eta_{2 \delta\left(g_{2 j}\right)}^{(j)}}=$ 1.

Theorem 26 can be directly deduced from Theorem 18 and thus its proof is omitted.

\section{Developed VIKOR Method with BDHF Information}

In this section, we introduce the proposed method, mainly including the modeling of MADA problems under BDHF environment, determination of attribute weights, and the process of extended VIKOR method.

4.1. Modeling of MADA Problems with BDHF Information. Differing from conventional MADA approaches, fuzzy decision matrix with BDHF assessments is constructed in this section. 
Suppose that a MADA problem includes $m$ alternatives $A_{i}(i=1, \ldots, m)$ and $n$ attributes $C_{j}(j=1, \ldots, n)$. The relative weights of $n$ attributes are represented by $w=\left(w_{1}\right.$, $\left.\ldots, w_{n}\right)^{T}$ such that $0 \leq w_{j} \leq 1(j=1, \ldots, n)$ and $\sum_{j=1}^{n} w_{j}=1$, where the notation " $T$ " denotes "transpose." Let the BDHF assessment of alternative $A_{i}$ on attribute $C_{j}$ be symbolized by $A_{i j}=\left(h_{i j}, g_{i j}\right)$. Given $h_{i j}=\left\{\left(\gamma_{i k}^{(j)}, p_{\gamma_{i k}^{(j)}}\right), i=1, \ldots, m ; j=1\right.$, $\left.\ldots, n, k=1, \ldots, \delta\left(h_{i j}\right)\right\}$ and $g_{i j}=\left\{\left(\eta_{i \tau}^{(j)}, p_{\eta_{i \tau}^{(j)}}\right), i=1, \ldots, m\right.$; $\left.j=1, \ldots, n, \tau=1, \ldots, \delta\left(g_{i j}\right)\right\}$ such that $\gamma_{i k}^{(j)} \in[0,1], p_{\gamma_{i k}^{(j)}} \in$ $[0,1], \eta_{i \tau}^{(j)} \in[0,1], p_{\eta_{i \tau}^{(j)}} \in[0,1], \gamma_{i k}^{(j)}+\eta_{i \tau}^{(j)} \leq 1$, and $p_{\gamma_{i k}^{(j)}} \times p_{\eta_{i \tau}^{(j)}} \leq$ 1 , then a BDHF decision matrix can be profiled by

$$
A_{m \times n}=\left[\begin{array}{cccc}
\left\langle h_{11}, g_{11}\right\rangle & \left\langle h_{12}, g_{12}\right\rangle & \cdots & \left\langle h_{1 n}, g_{1 n}\right\rangle \\
\left\langle h_{21}, g_{21}\right\rangle & \left\langle h_{22}, g_{22}\right\rangle & \cdots & \left\langle h_{2 n}, g_{2 n}\right\rangle \\
\vdots & \vdots & \ddots & \vdots \\
\left\langle h_{m 1}, g_{m 1}\right\rangle & \left\langle h_{m 2}, g_{m 2}\right\rangle & \cdots & \left\langle h_{m n}, g_{m n}\right\rangle
\end{array}\right] \text {. }
$$

It can be known from Definition 7 that each element in $A_{m \times n}$ is a BDHF element, so $A_{m \times n}$ can be regarded as a matrix of BDHF elements.

4.2. Determination of Attribute Weights. Weight, as a useful technique for reflecting relative importance of objectives, has been widely applied in MADA. To date, a lot of methods for determining weights are proposed such as AHP, maximizing deviation method, the CRITIC (criteria importance through intercriteria correlation) method [32], the standard deviation method [32], and especially entropy method which has been extensively used in fuzzy set. Motivated by AIF entropy measure, we define a new entropy measure with BDHF information.

Definition 27. Let $A_{i j}=\left(h_{i j}, g_{i j}\right)=\left\{\left(\gamma_{i k}^{(j)}, p_{\gamma_{i k}^{(j)}}\right),\left(\eta_{i \tau}^{(j)}\right.\right.$, $\left.\left.p_{\eta_{i \tau}^{(j)}}\right)\right\}\left(i=1, \ldots, m, j=1, \ldots, n, k=1, \ldots, \delta\left(h_{i j}\right), \tau=1\right.$, $\left.\ldots, \delta\left(g_{i j}\right)\right)$ and $w=\left(w_{1}, \ldots, w_{n}\right)^{T}$ be a BDHF decision matrix and attribute weights for a MADA problem, respectively. Then, entropy measure $E_{j}$ with BDHF information is defined as follows:

$$
E_{j}=-\frac{1}{m \ln 2} \sum_{i=1}^{m}\left[\sum_{k=1}^{\delta\left(h_{i j}\right)} \sum_{\tau=1}^{\delta\left(g_{i j}\right)} p_{\gamma_{i k}^{(j)}} p_{\eta_{i \tau}^{(j)}}\left(\gamma_{i k}^{(j)} \ln \gamma_{i k}^{(j)}+\eta_{i \tau}^{(j)} \ln \eta_{i \tau}^{(j)}-\left(\gamma_{i k}^{(j)}+\eta_{i \tau}^{(j)}\right) \ln \left(\gamma_{i k}^{(j)}+\eta_{i \tau}^{(j)}\right)-\left(1-\gamma_{i k}^{(j)}-\eta_{i \tau}^{(j)}\right) \ln 2\right)\right]
$$

where $\delta(h)$ and $\delta(g)$ symbolize the numbers of possible membership and nonmembership degrees, respectively.

Through observing the features of entropy measure in Definition 27, it can be inferred that entropy measure $E_{j}$ could be considered a compound function with respect to possible membership degree $\gamma_{i k}^{(j)}$ and possible nonmembership degree $\eta_{i \tau}^{(j)}$. In order to obtain axiomatic conditions of the entropy measure, a rule about the mentioned compound function in Theorem 28 is firstly demonstrated.

Theorem 28. Given a function $E(x, y)=-(1 / \ln 2)(x \ln x+$ $y \ln y-(x+y) \ln (x+y)-(1-x-y) \ln 2)$, where $x, y \in[0,1]$, it satisfies that

(1) when $x \leq y, E(x, y)$ is increasing with respect to $x$ and decreasing for $y$;

(2) when $x \geq y, E(x, y)$ is increasing with respect to $y$ and decreasing for $x$.

Theorem 28 is proven in Section A.4 of Appendix A.

Based on Definition 27 and Theorem 28, it can be verified that the BDHF entropy measure satisfies the following axiomatic conditions.

Theorem 29. A real function $E: B D H F$ sets $(X) \rightarrow[0,1]$ is called a BDHF entropy if $E$ satisfies the following axiomatic requirements:

(1) $E_{j}=0$ if each BDHF set $A \in 2^{x}$ (A, nonfuzzy).
(2) $E_{j}=1$ if $\gamma_{i k}^{(j)}=\eta_{i \tau}^{(j)}$ for all $i=1, \ldots, m, k=1, \ldots$, $\delta\left(h_{i j}\right), \tau=1, \ldots, \delta\left(g_{i j}\right)$.

(3) $E_{1} \leq E_{2}$ if, when $p_{\gamma_{i k}^{(1)}}=p_{\gamma_{i k}^{(2)}}$ and $p_{\eta_{i \tau}^{(1)}}=p_{\eta_{i \tau}^{(2)}}, \gamma_{i k}^{(1)} \leq$ $\gamma_{i k}^{(2)} \leq \eta_{i \tau}^{(2)} \leq \eta_{i \tau}^{(1)}$ or $\gamma_{i k}^{(1)} \geq \gamma_{i k}^{(2)} \geq \eta_{i \tau}^{(2)} \geq \eta_{i \tau}^{(1)}$.

(4) $E(A)=E\left(A^{c}\right)$.

Theorem 29 is proven in Section A.5 of Appendix A with the help of Theorem 28.

After obtaining the new entropy measure, the weight of attribute $C_{j}(j=1, \ldots, n)$ for alternative $A_{i}(i=1, \ldots, m)$ is determined by

$$
w_{j}=\frac{1-E_{j}}{n-\sum_{j=1}^{n} E_{j}},
$$

where $w_{j} \in[0,1], \sum_{j=1}^{n} w_{j}=1$, and $E_{j}$ can be calculated by (24).

According to entropy theory, the smaller the entropy value for each attribute is, the more the useful information the decision maker can acquire. Therefore, the attribute should be assigned a bigger weight and vice versa.

4.3. Procedure of the Developed VIKOR Method. VIKOR method is used to find the compromise solution which is the closest to the ideal solution, and a compromise means an agreement established by mutual concessions [14, 15]. So far, it has been widely used in solving decision making with conflicting and noncommensurable attributes in 


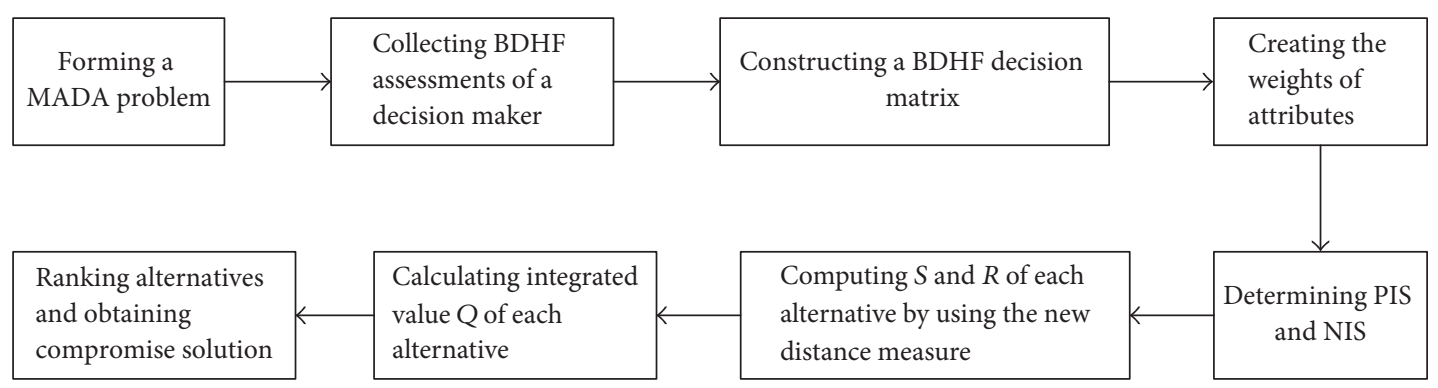

FIgURE 1: The procedure of the proposed method.

MADA. Kim and Chung proposed a fuzzy VIKOR approach to assess the vulnerability of the water supply to climate change and variability in South Korea [33]. Under interval type 2 fuzzy environment, Qin et al. proposed an extended VIKOR method based on prospect theory for multiple attribute decision making [12]. Based on HF set, an extended VIKOR method is developed by Zhang and Wei to solve multiple attribute decision making problems [34]. It can be deduced from the mentioned references that there are three potential hypotheses in fuzzy VIKOR method for solving MADA problems: (1) it is difficult to express the preference of a decision maker exactly; (2) there are conflicting and noncommensurable attributes in MADA; and (3) decision makers can accept compromise solutions.

Then, the procedure of the VIKOR method in BDHF context to deal with MADA problems is shown in Figure 1, which will be elaborated step by step.

Step 1. Identify $n$ attributes and $m$ alternatives to form a MADA problem.

Step 2. Construct a BDHF decision matrix in which BDHF assessments are provided by a decision maker.

Step 3. Create attribute weights based on the extended entropy measure.

Step 4. Determine the positive ideal solution (PIS) $A_{j}^{+}$and the negative ideal solution (NIS) $A_{j}^{-}$in each attribute $j=$ $1,2, \ldots, n$. If the $j$ th attribute represents a benefit, it can be acquired that

$$
\begin{array}{ll}
A_{j}^{+}=(\{1,1\},\{0,1\}), & (j=1, \ldots, n), \\
A_{j}^{-}=(\{0,1\},\{1,1\}), \quad(j=1, \ldots, n) .
\end{array}
$$

Step 5. Compute the values $S_{i}$ and $R_{i}, i=1,2, \ldots, m$, by the following equations:

$$
\begin{aligned}
& S_{i}=\sum_{j=1}^{n} \frac{w_{j} d\left(A_{i j}, A_{j}^{+}\right)}{d\left(A_{j}^{+}, A_{j}^{-}\right)}, \\
& R_{i}=\max _{j} \frac{w_{j} d\left(A_{i j}, A_{j}^{+}\right)}{d\left(A_{j}^{+}, A_{j}^{-}\right)},
\end{aligned}
$$

where $w_{j}$ is the weight of attribute, satisfying $\sum_{j=1}^{n} w_{j}=1$, which can be obtained from (23).

Step 6. Calculate the values $Q_{i}, i=1,2, \ldots, m$, using the following equation:

$$
Q_{i}=\frac{v\left(S_{i}-S^{-}\right)}{\left(S^{+}-S^{-}\right)}+\frac{(1-v)\left(R_{i}-R^{-}\right)}{\left(R^{+}-R^{-}\right)},
$$

where $S^{+}=\max _{i} S_{i}, S^{-}=\min _{i} S_{i}, R^{+}=\max _{i} R_{i}$, and $R^{-}=$ $\max _{i} R_{i}$. The parameter $v \in[0,1]$ is introduced as a weight of the strategy of total satisfaction, where $1-v$ is the weight of the individual regret. Here, $v$ is a balance parameter between total satisfaction and individual regret. Without loss of generality, the value of $v$ is set to 0.5 [34-37]. From (27), it can be deduced that the value of $v$ will influence the value of $Q_{i}$ and further may influence the final ranking order.

Step 7. Rank the alternatives, sorting by the values $S_{i}, R_{i}$, and $Q_{i}$ along with increasing order. The results are three ranking lists: $\{A\}_{S},\{A\}_{R}$, and $\{A\}_{Q}$. The index $Q_{i}$ implies the separation measure of $A_{i}$ from the best alternative. That is, the smaller the value $Q$ is obtained, the better the alternative will be.

Step 8. Propose a compromise solution in which the alternative $\left(A^{(1)}\right)$ is the best ranked by the measure $Q$ (minimum) if the following two conditions are satisfied.

\section{(C1) Acceptable Advantage}

$$
Q\left(A^{(2)}\right)-Q\left(A^{(1)}\right) \geq D Q
$$

where $A^{(2)}$ is the alternative with second position in the ranking list $\{A\}_{Q}, D Q=1 /(m-1)$, where $m$ indicates the number of feasible alternatives.

(C2) Acceptable Stability in Decision Making. The alternative $A^{(1)}$ must also be the best ranked by $S$ or/and $R$. This compromise solution is stable within a decision making process, which could be as follows: "voting by majority rule" (where $v>0.5$ is needed) or "by consensus" $(v \approx 0.5)$ or "with veto" $(v<0.5)$. Here, $v$ is the weight of the decision making strategy "the maximum group utility."

If one of these conditions is not satisfied, then a set of compromise solutions is proposed, which consists of 
(1) alternative $A^{(1)}$ and $A^{(2)}$ if only condition (C2) is not satisfied; or

(2) alternative $A^{(1)}, A^{(2)}, \ldots, A^{(M)}$ if condition (C1) is not satisfied; $A^{(M)}$ is determined by the relation $Q\left(A^{(M)}\right)$ $Q\left(A^{(1)}\right)<D Q$ for maximum $M$ (the positions of these alternatives are "in closeness").

\section{An Application of the Proposed Method}

In this section, an assessment problem of people's livelihood project is analyzed by the proposed fuzzy VIKOR method to demonstrate its validity and applicability.

5.1. Description of People's Livelihood Project. Premier Li in China, on behalf of the State Council, in the Report on the Work of the Government 2015 pointed out that part of the important work of the government in 2015 was to strengthen safeguards for people's standard of living which is the basis of achieving steady and sound economic development and ensuring social harmony and stability. Establishing people's livelihood project is the product of the mentioned work. Here, people's livelihood is a concept reflecting specific characteristic of China. The regions which have different performances in people's livelihood project can be considered to be assigned different resources and opportunities to enhance their development. So, how to assess people's livelihood of different regions is a hot topic attracting wide range of attention, which can be regarded as a MADA problem.

In this section, taking seven regions in East China as an example, we investigate how to help a decision maker which is an official from Ministry of Civil Affairs of the People's Republic of China to select several relatively better ones of these regions to be demonstration zones which can be given top priority to obtain resources and support. The decision maker firstly identifies seven regions including Shanghai $\left(A_{1}\right)$, Jiangsu $\left(A_{2}\right)$, Zhejiang $\left(A_{3}\right)$, Anhui $\left(A_{4}\right)$, Fujian $\left(A_{5}\right)$, Jiangxi $\left(A_{6}\right)$, and Shandong $\left(A_{7}\right)$. Based on the previous research [38], seven attributes are selected including income and employment $\left(C_{1}\right)$, public finance income $\left(C_{2}\right)$, welfare guarantee $\left(C_{3}\right)$, health $\left(C_{4}\right)$, culture and education $\left(C_{5}\right)$, residence and transportation $\left(C_{6}\right)$, and environmental protection $\left(C_{7}\right)$. Hereinto, the preference of the decision maker is considered neutrality between total satisfaction and individual regret by interviewing with him. Thus, the parameter $v$ in this assessment problem is set to 0.5. Owing to the limitation of knowledge and experience, the decision maker find difficulty in providing scientific attribute weights. Thus, the weights of attributes will be determined by an objective method in this problem. Step 1 is completed.

In order to help the decision maker generate BDHF assessments, five experts are invited from Department of Housing and Urban-Rural Development of Anhui Province, Department of Civil Affairs of Anhui Province, and Hefei University of Technology to independently and anonymously evaluate the seven regions on each attribute. Then, the decision maker combines the experts' opinions and his preference to provide a BDHF decision matrix $A_{7 \times 7}$, as shown in Table 1 . Step 2 is completed.
5.2. Solution to the Assessment Problem. Due to the nature of the problem of evaluating people's livelihood projects, there is no information available for the decision maker to subjectively assign attribute weights. As a result, the entropy method in Section 4.2 is applied to objectively determine attribute weights for the problem. By using (22)-(23), the weights of the seven attributes in the problem are created, which are $w_{j}(j=$ $1, \ldots, 7)=(0.138,0.150,0.148,0.163,0.165,0.107,0.127)^{T}$. Step 3 is completed. From Section 4.3, we can obtain that the positive ideal solution and the negative ideal solution are set to $A_{j}^{+}=(\{1,1\},\{0,1\}), A_{j}^{-}=(\{0,1\},\{1,1\})(j=1, \ldots, 7)$ so as to simplify complexity of computing. Step 4 is completed.

When the attribute weights for the problem are known, the distance between each alternative and positive ideal solution and that between positive and negative ideal solutions can be measured and further applied to generate $S_{i}(i=$ $1, \ldots, 7)$ and $R_{i}(i=1, \ldots, 7)$. Using Step 4 , it can be obtained that the distance measure between positive and negative ideal solutions is still equal to one. Thus, the distance measure between each alternative and positive ideal solution can be calculated in Table 2. Based on (26), we can then obtain $S_{i}(i=1, \ldots, 7)=(0.295,0.286,0.312,0.454,0.511$, $0.459,0.323)$ and $R_{i}(i=1, \ldots, 7)=(0.058,0.055,0.062$, $0.091,0.099,0.082,0.063)$. Step 5 is completed.

Because the parameter $v$ equals 0.5 , it can be calculated from $S_{i}(i=1, \ldots, 7)$ and $R_{i}(i=1, \ldots, 7)$ that $Q_{i}(i=1$, $\ldots, 7)=(0.028,0.017,0.142,0.787,1,0.685,0.167)$. Then, three ranking orders can be generated in Table 3 by $S_{i}(i=$ $1, \ldots, 7), R_{i}(i=1, \ldots, 7)$, and $Q_{i}(i=1, \ldots, 7)$, respectively. Although the ranking order of seven regions by $Q_{i}(i=$ $1, \ldots, 7)$ can be obtained to select the best performing one, the final purpose of this problem has not been achieved. That is, we need to select a set of compromise solutions to be demonstration zones. Steps 6 and 7 are completed.

In order to further obtain compromise solution, the two conditions in Step 8 of the procedure of VIKOR method are introduced. In Table 3, it is easy to notice that the value of $Q_{2}$ is the smallest which can be considered the best alternative and the ranking order by $Q_{i}(i=1, \ldots, 7)$ is $A_{2}>A_{1}>$ $A_{3}>A_{7}>A_{6}>A_{4}>A_{5}$, where the notation " $>$ " means "prior to." Then, based on the mentioned two conditions in Step 8, it can be calculated that $Q_{1}-Q_{2}=0.028-0.017=$ $0.011 \leq 1 /(7-1), Q_{3}-Q_{2}=0.142-0.017=0.125 \leq$ $1 /(7-1), Q_{7}-Q_{2}=0.167-0.017=0.15 \leq 1 /(7-1)$, and $Q_{6}-Q_{2}=0.685-0.017=0.668 \geq 1 /(7-1)$. Thus, we can state that a set of compromise solutions includes $A_{1}, A_{2}, A_{3}$, and $A_{7}$. That is, the four regions including Shanghai, Jiangsu, Zhejiang, and Shandong in the above compromise solution can be preferentially developed, which are considered almost the same from the viewpoint of the decision maker. Step 8 is completed.

From Table 3, it can be seen that the ranking order of alternatives is changing as the value of parameter $v$ varies from 0 to 1 . When $v=1$, the optimal alternative has been $A_{1}$ instead of $A_{2}$. When $v=0$, a compromise solution includes all alternatives by using Step 8 . Thus, parameter $v$ is considered attitude of the decision maker to balance total satisfaction and individual regret. 


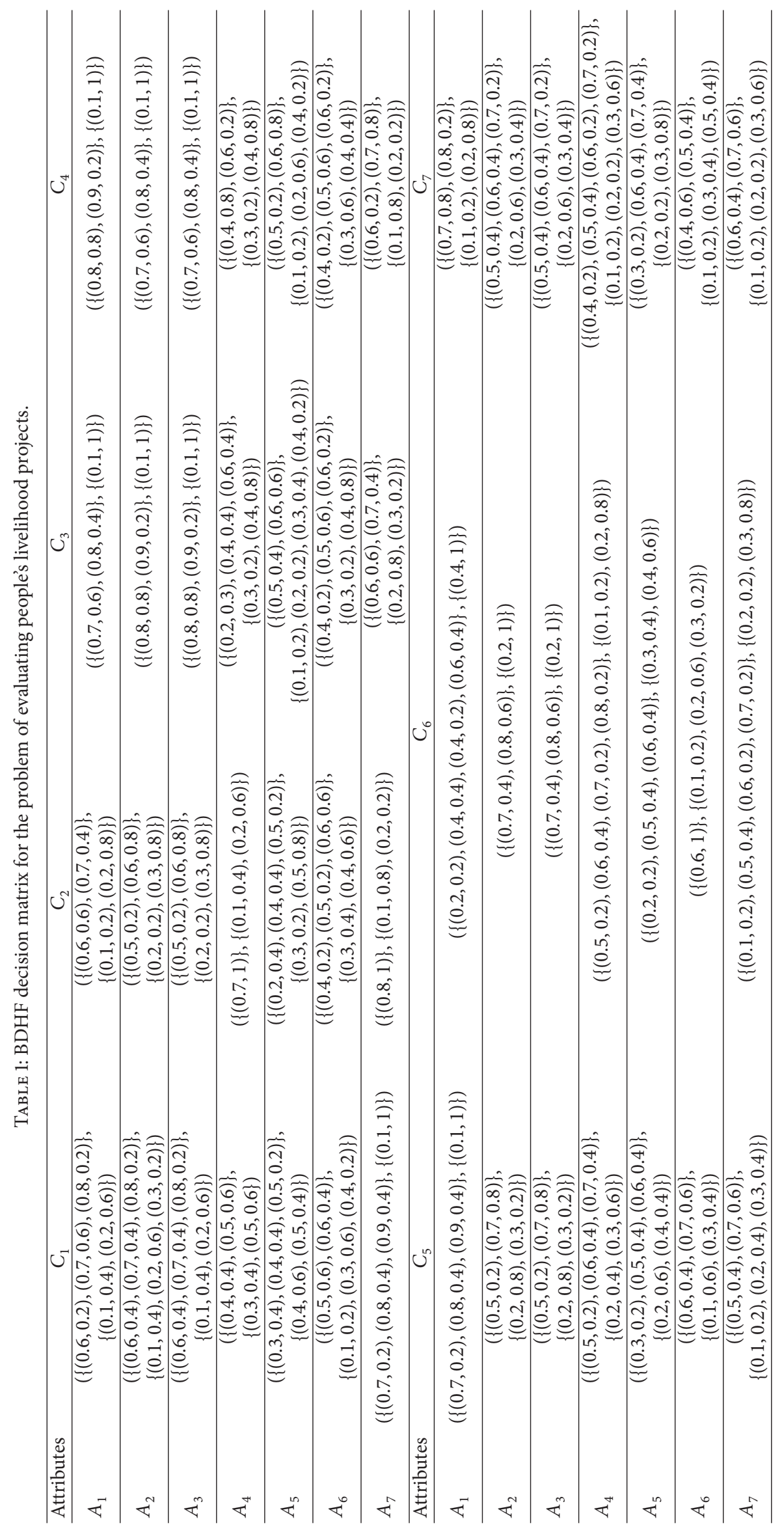


TABLE 2: Distance measure between each alternative and positive ideal solution on each attribute.

\begin{tabular}{lccccccc}
\hline Attributes & $C_{1}$ & $C_{2}$ & $C_{3}$ & $C_{4}$ & $C_{5}$ & $C_{6}$ & $C_{7}$ \\
\hline$d\left(A_{1 j}, A^{+}\right)$ & 0.3 & 0.36 & 0.26 & 0.18 & 0.18 & 0.54 & 0.28 \\
$d\left(A_{2 j}, A^{+}\right)$ & 0.32 & 0.24 & 0.3 & 0.34 & 0.28 & 0.32 & 0.26 \\
$d\left(A_{3 j}, A^{+}\right)$ & 0.32 & 0.42 & 0.18 & 0.26 & 0.34 & 0.24 & 0.42 \\
$d\left(A_{4 j}, A^{+}\right)$ & 0.54 & 0.3 & 0.56 & 0.56 & 0.38 & 0.36 & 0.46 \\
$d\left(A_{5 j}, A^{+}\right)$ & 0.62 & 0.66 & 0.44 & 0.42 & 0.5 & 0.52 & 0.42 \\
$d\left(A_{6 j}, A^{+}\right)$ & 0.46 & 0.46 & 0.5 & 0.5 & 0.34 & 0.4 & 0.56 \\
$d\left(A_{7 j}, A^{+}\right)$ & 0.18 & 0.2 & 0.36 & 0.32 & 0.38 & 0.52 & 0.34 \\
\hline
\end{tabular}

TABLE 3: The ranking order of the regions by $S, R$, and $Q$.

\begin{tabular}{lccccccc}
\hline Attributes & $A_{1}$ & $A_{2}$ & $A_{3}$ & $A_{4}$ & $A_{5}$ & $A_{6}$ & $A_{7}$ \\
\hline By $S$ & 0.287 & 0.295 & 0.312 & 0.454 & 0.511 & 0.459 & 0.323 \\
Ranking & 1 & 2 & 3 & 5 & 7 & 6 & 4 \\
By $R$ & 0.058 & 0.055 & 0.062 & 0.091 & 0.099 & 0.082 & 0.063 \\
Ranking & 2 & 1 & 3 & 6 & 7 & 5 & 4 \\
By $Q$ & 0.028 & 0.017 & 0.142 & 0.787 & 1 & 0.685 & 0.167 \\
Ranking & 2 & 1 & 3 & 6 & 7 & 5 & 4 \\
\hline
\end{tabular}

5.3. Comparison with TOPSIS Method. Similar to VIKOR method, TOPSIS (technique for order preference by similarity to ideal solution) is also a distance-based method. TOPSIS method proposed by Hwang and Yoon [39] can determine a solution with the shortest distance to the positive ideal solution and the farthest distance to the negative ideal solution. Many researchers had compared these two methods in different contexts such as crisp numbers [14], hesitant fuzzy elements [34], and interval type 2 fuzzy numbers [12]. In this section, the two methods will be compared in BDHF environment. Then, the main process of the TOPSIS method instead of the VIKOR method is demonstrated in the following. Here, some steps which are similar to VIKOR method are omitted, such as the construction of decision matrix.

Step 1. Determine the positive ideal solution $A^{+}$and negative ideal solution $A^{-}$for BDHF decision matrix in (21), respectively.

$$
\begin{aligned}
& A^{+}=\left\{A_{1}^{+}, \ldots, A_{n}^{+}\right\}, \\
& A^{-}=\left\{A_{1}^{-}, \ldots, A_{n}^{-}\right\},
\end{aligned}
$$

where

$$
\begin{array}{ll}
A_{j}^{+} & =(\{1,1\},\{0,1\}), \quad(j=1, \ldots, 7), \\
A_{j}^{-} & =(\{0,1\},\{1,1\}), \quad(j=1, \ldots, 7) .
\end{array}
$$

Step 2. Calculate the separation measures using the proposed distance measure in Definition 13.

$$
\begin{array}{r}
d\left(A_{i}, A^{+}\right)=\sum_{j=1}^{n} w_{j} \cdot d\left(A_{i j}, A_{j}^{+}\right) \\
=(0.286,0.293,0.311,0.453,0.510,0.458,0.322), \\
(i=1, \ldots, 7),
\end{array}
$$

$$
\begin{array}{r}
d\left(A_{i}, A^{-}\right)=\sum_{j=1}^{n} w_{j} \cdot d\left(A_{i j}, A_{j}^{-}\right) \\
=(0.836,0.783,0.815,0.705,0.674,0.699,0.817), \\
(i=1, \ldots, 7) .
\end{array}
$$

Step 3. Calculate the closeness coefficient of each alternative.

$$
\begin{aligned}
\mathrm{CC}_{i} & =\frac{d\left(A_{i}, A^{-}\right)}{d\left(A_{i}, A^{-}\right)+d\left(A_{i}, A^{+}\right)} \\
& =(0.749,0.727,0.724,0.609,0.569,0.604,0.718),
\end{aligned}
$$

$$
(i=1, \ldots, 7) .
$$

Step 4. Rank the alternatives through the value of closeness coefficient as $A_{1}>A_{2}>A_{3}>A_{7}>A_{4}>A_{6}>A_{5}$, where the notation " $>$ " means "prior to."

The ranking order of alternatives using VIKOR method is slightly different from that using TOPSIS method, especially in the selection of the appropriate alternatives. In the TOPSIS method, a solution is selected with the shortest distance from the positive ideal solution and the farthest distance from the negative ideal solution, while VIKOR method focuses on finding a compromise solution considering the balance between total satisfaction and individual regret. As the TOPSIS method ignores the relative importance of the mentioned distances [7,34], it is more suitable for a decision maker whose risk attitude is risk-aversion. Compared with TOPSIS method, VIKOR considers the relative importance of the distance to the ideal solutions during the process of calculating comprehensive ranking index. Furthermore, in some situations it is found that VIKOR is more robust than TOPSIS when assessments are changed [13]. That is another reason why we are concerned about VIKOR in this paper. Meanwhile, the result generated by TOPSIS method is a ranking order of all alternatives. The decision maker can select an optimum solution but it is difficult to obtain compromise solution. In a real case, it is always necessary to obtain a compromise solution including several alternatives instead of an optimal solution. Compared with TOPSIS method, VIKOR can avoid this drawback and is better used in solving MADA problems to reach a compromise solution $[7,34]$.

Thus, from the application of the proposed method in evaluating people's livelihood project of several regions and the above comparative analysis, the main advantages of the proposed method are demonstrated as follows from both practical and theoretical perspectives:

(1) It proposes BDHF set to help a decision maker express their preference in solving a MADA problem which is more objective and scientific than many existing fuzzy sets. From the practical perspective, BDHF set is more suitable in solving a practical MADA problem such as the evaluation of people's livelihood project of several regions in China. 
(2) It defines a new generalized distance measure between BDHF assessments to avoid missing or changing much original information provided by the decision maker, which is more suitable for the most real-world decision making, for example, the evaluation problem of people's livelihood project of several regions in China.

(3) It determines attribute weights by using a new entropy measure with BDHF information, which can avoid subjective and arbitrary judgment of the decision maker.

(4) It generates a compromise solution instead of an optimum solution to help the decision maker achieve decision goal, which is more suitable for addressing a practical MADA problem, such as the evaluation problem of people's livelihood project of several regions in China.

In addition, the complexity of the proposed method is discussed from the following three aspects: (1) in the process of collecting the preferences of a decision maker, BDHF set is easier to be used by him/her to cover more information than other expression forms; (2) an entropy measure with BDHF information is proposed to objectively determine attribute weights, which can relieve the burden of the decision maker; and (3) the proposed distance measure relaxes the potential conditions of the most existing distance measures and avoids determining an exact value of parameter $\lambda$ by the decision maker, which can further relieve the burden of the decision maker.

Overall, the proposed method is easy to be implemented in solving practical MADA problems.

\section{Conclusion}

Combining the advantages of AIF information with that of HF information, BDHF information is defined in this paper to present the preferences of a decision maker or an expert. In order to solve MADA problems with BDHF information, a new VIKOR method is proposed. Here, attribute weights are determined by a new entropy measure with BDHF information. In VIKOR method, the definition of distance measure is a key step. However, the effect of parameter $\lambda$ in distance measure has been a controversial issue. Thus, based on AIF distance measure and HF distance measure, a generalized distance measure between two BDHF elements is designed where all possible values of parameter $\lambda$ are considered. Finally, the proposed VIKOR method is applied to evaluate people's livelihood project in seven regions of East China to select several regions where the performances of people's livelihood project are relatively better. Meanwhile, the VIKOR method is compared with the TOPSIS method to further show the advantages of the proposed method.

Although the proposed method in this paper provides a way to find solutions to MADA problems with BDHF assessments, it cannot analyze more complex problems where interval-valued BDHF assessments are given by a group of decision makers. To address such challenge, we intend to extend the method to analyze group decision making problems with interval-valued BDHF assessments.

\section{Appendix}

\section{A. Proofs}

A.1. Proof of Theorem 18. It can be deduced from Definition 13 that $d\left(\alpha_{1}, \alpha_{2}\right)=\sum_{i=1}^{\delta\left(h_{1}\right)} \sum_{j=1}^{\delta\left(g_{1}\right)} \sum_{p=1}^{\delta\left(h_{2}\right)} \sum_{q=1}^{\delta\left(g_{2}\right)} p_{\gamma_{1 i}} p_{\gamma_{2 p}} p_{\eta_{1 j}} p_{\eta_{2 q}}(0.5$. $\left.\left(\left(\gamma_{1 i}-\gamma_{2 p}\right)^{\lambda}+\left(\eta_{1 j}-\eta_{2 q}\right)^{\lambda}\right)\right)^{1 / \lambda}$. Because $1 \leq \lambda \leq+\infty, 0 \leq$ $\gamma_{1 i}, \gamma_{2 p}, \eta_{1 j}, \eta_{2 q} \leq 1,0 \leq \gamma_{1 i}+\eta_{1 j} \leq 1$, and $0 \leq \gamma_{2 p}+\eta_{2 q} \leq 1$, we can verify that $0 \leq 0.5\left(\left(\gamma_{1 i}-\gamma_{2 p}\right)^{\lambda}+\left(\eta_{1 j}-\eta_{2 q}\right)^{\lambda}\right)^{1 / \lambda} \leq 1$. Moreover, it can be obtained that $d\left(\alpha_{1}, \alpha_{2}\right) \leq$ $\sum_{i=1}^{\delta\left(h_{1}\right)} \sum_{j=1}^{\delta\left(g_{1}\right)} \sum_{p=1}^{\delta\left(h_{2}\right)} \sum_{q=1}^{\delta\left(g_{2}\right)} p_{\gamma_{1 i}} p_{\gamma_{2 p}} p_{\eta_{1 j}} p_{\eta_{2 q}}=\left(p_{\gamma_{11}}+\cdots+\right.$ $\left.p_{\gamma_{1 \delta\left(h_{1}\right)}}\right)\left(p_{\gamma_{21}}+\cdots+p_{\gamma_{2 \delta\left(h_{2}\right)}}\right)\left(p_{\eta_{11}}+\cdots+p_{\eta_{1 \delta\left(g_{1}\right)}}\right)\left(p_{\eta_{21}}+\cdots+p_{\left.\eta_{2 \delta(g)}\right)}\right)=$ $\sum_{i=1}^{\delta\left(h_{1}\right)} p_{\gamma_{1 i}} \cdot \sum_{p=1}^{\delta\left(h_{2}\right)} p_{\gamma_{2 p}} \cdot \sum_{j=1}^{\delta\left(g_{1}\right)} p_{\eta_{1 j}} \cdot \sum_{q=1}^{\delta\left(g_{2}\right)} p_{\eta_{2 q}}$

Besides, it can be inferred that $\sum_{i=1}^{\delta\left(h_{1}\right)} p_{\gamma_{1 i}}=1, \sum_{p=1}^{\delta\left(h_{2}\right)} p_{\gamma_{2 p}}=$ $1, \sum_{j=1}^{\delta\left(g_{1}\right)} p_{\eta_{1 j}}=1, \sum_{q=1}^{\delta\left(g_{2}\right)} p_{\eta_{2 q}}=1$.

Thus, property (1) holds.

Definition 13 indicates that property (2) clearly holds.

If $\delta\left(h_{1}\right)=\delta\left(h_{2}\right)=\delta\left(g_{1}\right)=\delta\left(g_{2}\right)=1, \gamma_{11}=\gamma_{21}, \eta_{11}=$ $\eta_{21}$, and $p_{\gamma_{11}}=p_{\gamma_{21}}=p_{\eta_{11}}=p_{\eta_{21}}=1$, then $\alpha_{1}$ and $\alpha_{2}$ reduce to two equivalent AIF numbers. It is obvious that $d\left(\alpha_{1}, \alpha_{2}\right)=0$. Property (3) is proven.

A.2. Proof of Lemma 21. Suppose that $a \geq b$. For $F(\lambda)$, it can be obtained that

$$
\begin{aligned}
\frac{d F}{d \lambda}= & \left(\frac{a^{\lambda}+b^{\lambda}}{2}\right) \\
& \cdot\left(\frac{a^{\lambda} \ln a+b^{\lambda} \ln b}{\lambda\left(a^{\lambda}+b^{\lambda}\right)}-\frac{1}{\lambda^{2}} \ln \left(\frac{a^{\lambda}+b^{\lambda}}{2}\right)\right) \\
= & \left(\frac{a^{\lambda}+b^{\lambda}}{2 \lambda}\right) \\
& \cdot\left(\frac{a^{\lambda} \ln a+b^{\lambda} \ln b}{a^{\lambda}+b^{\lambda}}-\frac{1}{\lambda} \ln \left(\frac{a^{\lambda}+b^{\lambda}}{2}\right)\right) .
\end{aligned}
$$

Because $\left(a^{\lambda}+b^{\lambda}\right) / 2 \lambda>0$, in the following we only consider the value of $\left(a^{\lambda} \ln a+b^{\lambda} \ln b\right) /\left(a^{\lambda}+b^{\lambda}\right)-(1 / \lambda) \ln \left(\left(a^{\lambda}+b^{\lambda}\right) / 2\right)$. Suppose that $Q(b)=\left(a^{\lambda} \ln a+b^{\lambda} \ln b\right) /\left(a^{\lambda}+b^{\lambda}\right)-(1 / \lambda) \ln \left(\left(a^{\lambda}+\right.\right.$ $\left.b^{\lambda}\right) / 2$ ) such that $1 \leq \lambda \leq+\infty$ and $0 \leq a \leq 1$. Then, it can be inferred that

$$
\begin{aligned}
\frac{d Q}{d b} & =\frac{\lambda b^{\lambda-1} \ln b+b^{\lambda-1}}{a^{\lambda}+b^{\lambda}}-\frac{\lambda b^{\lambda-1}\left(a^{\lambda} \ln a+b^{\lambda} \ln b\right)}{\left(a^{\lambda}+b^{\lambda}\right)^{2}} \\
& -\frac{b^{\lambda-1}}{a^{\lambda}+b^{\lambda}}=\frac{b^{\lambda-1}}{\left(a^{\lambda}+b^{\lambda}\right)^{2}} \cdot\left((\lambda \ln b+1)\left(a^{\lambda}+b^{\lambda}\right)\right. \\
& \left.-\lambda\left(a^{\lambda} \ln a+b^{\lambda} \ln b\right)-\left(a^{\lambda}+b^{\lambda}\right)\right)=\lambda \cdot a^{\lambda} \\
& \cdot(\ln b-\ln a) .
\end{aligned}
$$


On the assumption that $a \geq b$, it can be deduced from $d Q / d b \leq 0$ and $d Q / d b=0$ if $a=b$ that $Q(b) \geq$ 0 . Consequently, it can be obtained that $d F / d \lambda \geq 0$ and $d F / d \lambda=0$ if $a=b$, which further verifies that $F(\lambda)$ is increasing with respect to $\lambda$.

The assumption of $a \geq b$ can be directly used to reason that $\left(0.5\left(a^{\lambda}+b^{\lambda}\right)\right)^{1 / \lambda} \leq a$.

Therefore, Lemma 21 holds.

A.3. Proof of Theorem 23. From the discussion after Lemma 21, it can be obtained that $\lim _{\lambda_{0} \rightarrow+\infty} \int_{1}^{\lambda_{0}} f(\lambda) d \lambda$ and $\lim _{\lambda_{0} \rightarrow+\infty}\left(\lambda_{0}-1\right)$ are divergent and $\lim _{\lambda \rightarrow+\infty}\left(\left(\int_{1}^{\lambda} f(\lambda) d \lambda\right) d \lambda /\right.$ $(\lambda-1) d \lambda)$ exists.

Therefore, it can be inferred from L'Hopital's Rule in Lemma 22 that $\overline{f(\lambda)}=\lim _{\lambda_{0} \rightarrow+\infty}\left(\int_{1}^{\lambda_{0}} f(\lambda) d \lambda /\left(\lambda_{0}-1\right)\right)=$ $\lim _{\lambda \rightarrow+\infty}\left(\left(\int_{1}^{\lambda} f(\lambda) d \lambda\right) d \lambda /(\lambda-1) d \lambda\right)=\lim _{\lambda \rightarrow+\infty} f(\lambda) / 1=$ $\max (a, b)$.

Thus, Theorem 23 holds.

A.4. Proof of Theorem 28. Firstly, taking the partial derivative of $E(x, y)$ with respect to $x$ yields

$$
\frac{\partial E(x, y)}{\partial x}=-\frac{\ln (2 x /(x+y))}{\ln x}
$$

Secondly, one can find the critical point of $E(x, y)$ denoted by $x^{*}$ from the equation $\partial E(x, y) / \partial x=0$, which means that $x^{*}=y$. Therefore, for $x, y \in[0,1]$, it can be deduced that when $x \leq y, \partial E(x, y) / \partial x \geq 0$ and when $x \geq y$, $\partial E(x, y) / \partial x \leq 0$. Similarly, it can be acquired that when $x \leq y, \partial E(x, y) / \partial y \leq 0$ and when $x \geq y, \partial E(x, y) / \partial y \geq 0$.

Finally, Theorem 28 holds.

\section{A.5. Proof of Theorem 29}

(1) When each BDHF element reduced to a crisp set, it can be inferred that $h_{i j}=1$ and $g_{i j}=0$ or $h_{i j}=0$ and $g_{i j}=1$ for all $i=1, \ldots, m, j=1, \ldots, n$. Therefore, based on Definition 27, property (1) holds.

(2) If $\gamma_{i k}^{(j)}=\eta_{i \tau}^{(j)}$ for all $i=1, \ldots, m, k=1, \ldots, \delta\left(h_{i j}\right)$, $\tau=1, \ldots, \delta\left(g_{i j}\right)$, it can be deduced from Definition 27 that $E(A)=1$. Property (2) holds.

(3) Because $p_{\gamma_{i k}^{(1)}}=p_{\gamma_{i k}^{(2)}}$ and $p_{\eta_{i \tau}^{(1)}}=p_{\eta_{i \tau}^{(2)}}$, it can be obtained from Theorem 28 that when $\gamma_{i k}^{(1)} \leq \gamma_{i k}^{(2)} \leq$ $\eta_{i \tau}^{(2)} \leq \eta_{i \tau}^{(1)}, E_{1}$ is increasing with respect to $\gamma_{i k}^{(1)}$ and decreasing for $\eta_{i \tau}^{(1)}$ and $E_{2}$ is increasing with respect to $\gamma_{i k}^{(2)}$ and decreasing for $\eta_{i \tau}^{(2)}$. The similar situation happens to $\gamma_{i k}^{(1)} \geq \gamma_{i k}^{(2)} \geq \eta_{i \tau}^{(2)} \geq \eta_{i \tau}^{(1)}$. Thus, it can be inferred from $\gamma_{i k}^{(1)} \leq \gamma_{i k}^{(2)} \leq \eta_{i \tau}^{(2)} \leq \eta_{i \tau}^{(1)}$ or $\gamma_{i k}^{(1)} \geq \gamma_{i k}^{(2)} \geq$ $\eta_{i \tau}^{(2)} \geq \eta_{i \tau}^{(1)}$ that $E_{1} \leq E_{2}$. Property (3) holds.

(4) Definition 27 indicates that property (4) clearly holds.

Thus, Theorem 29 holds.

\section{Competing Interests}

The authors declare that they have no competing interests.

\section{Acknowledgments}

This research was supported by the Foundation for Innovative Research Groups of the Natural Science Foundation of China (no. 71521001), the National Key Basic Research Program of China (no. 2013CB329603), and the National Natural Science Foundation of China (nos. 71571060, 71201043, 71303073, 71501054, and 71131002).

\section{References}

[1] J. S. Dyer, P. C. Fishburn, R. E. Steuer, J. Wallenius, and S. Zionts, "Multiple criteria decision making, multiattribute utility theory: the next ten years," Management Science, vol. 38, no. 5, pp. 645654, 1992.

[2] A. Mardani, A. Jusoh, and E. K. Zavadskas, "Fuzzy multiple criteria decision-making techniques and applicationstwo decades review from 1994 to 2014," Expert Systems with Applications, vol. 42, no. 8, pp. 4126-4148, 2015.

[3] T.-Y. Chen, "Interval-valued intuitionistic fuzzy QUALIFLEX method with a likelihood-based comparison approach for multiple criteria decision analysis," Information Sciences, vol. 261, pp. 149-169, 2014.

[4] J. Xu and F. Shen, "A new outranking choice method for group decision making under Atanassov's interval-valued intuitionistic fuzzy environment," Knowledge-Based Systems, vol. 70, pp. 177-188, 2014.

[5] D. Joshi and S. Kumar, "Interval-valued intuitionistic hesitant fuzzy Choquet integral based TOPSIS method for multi-criteria group decision making," European Journal of Operational Research, vol. 248, no. 1, pp. 183-191, 2016.

[6] I. Mahdavi, N. Mahdavi-Amiri, A. Heidarzade, and R. Nourifar, "Designing a model of fuzzy TOPSIS in multiple criteria decision making," Applied Mathematics and Computation, vol. 206, no. 2, pp. 607-617, 2008.

[7] M. Yazdani and A. F. Payam, "A comparative study on material selection of microelectromechanical systems electrostatic actuators using Ashby, VIKOR and TOPSIS," Materials \& Design, vol. 65 , pp. 328-334, 2015.

[8] B. Zhu, Z. S. Xu, and M. M. Xia, "Hesitant fuzzy geometric Bonferroni means," Information Sciences, vol. 205, pp. 72-85, 2012.

[9] C. G. Li, S. Z. Zeng, T. J. Pan, and L. N. Zheng, "A method based on induced aggregation operators and distance measures to multiple attribute decision making under 2-tuple linguistic environment," Journal of Computer and System Sciences, vol. 80, no. 7, pp. 1339-1349, 2014.

[10] S. Opricovic, Multi-Criteria Optimization of Civil Engineering Systems, Faculty of Civil Engineering, Belgrade, Serbia, 1998.

[11] S. Opricovic and G.-H. Tzeng, "Multicriteria planning of postearthquake sustainable reconstruction," Computer-Aided Civil and Infrastructure Engineering, vol. 17, no. 3, pp. 211-220, 2002.

[12] J. D. Qin, X. W. Liu, and W. Pedrycz, "An extended VIKOR method based on prospect theory for multiple attribute decision making under interval type-2 fuzzy environment," KnowledgeBased Systems, vol. 86, pp. 116-130, 2015. 
[13] Y. Yuan, T. Guan, X.-B. Yan, and Y.-J. Li, "Based on hybrid VIKOR method decision making model for supplier selection," Control and Decision, vol. 29, no. 3, pp. 551-560, 2014 (Chinese).

[14] S. Opricovic and G.-H. Tzeng, "Compromise solution by MCDM methods: a comparative analysis of VIKOR and TOPSIS," European Journal of Operational Research, vol. 156, no. 2, pp. 445-455, 2004.

[15] S. Opricovic and G.-H. Tzeng, "Extended VIKOR method in comparison with outranking methods," European Journal of Operational Research, vol. 178, no. 2, pp. 514-529, 2007.

[16] Z. L. Yue, "An approach to aggregating interval numbers into interval-valued intuitionistic fuzzy information for group decision making," Expert Systems with Applications, vol. 38, no. 5, pp. 6333-6338, 2011.

[17] J. M. Merigo, D. Palacios-Marques, and S. Zeng, "Subjective and objective information in linguistic multi-criteria group decision making," European Journal of Operational Research, vol. 248, no. 2, pp. 522-531, 2016.

[18] J. L. Devore, Probability and Statistics for Engineering and the Sciences, Brooks/Cole, 6th edition, 2004.

[19] A. Shemshadi, H. Shirazi, M. Toreihi, and M. J. Tarokh, "A fuzzy VIKOR method for supplier selection based on entropy measure for objective weighting," Expert Systems with Applications, vol. 38, no. 10, pp. 12160-12167, 2011.

[20] B. Zhu and Z. S. Xu, "Hesitant fuzzy Bonferroni means for multi-criteria decision making," Journal of the Operational Research Society, vol. 64, no. 12, pp. 1831-1840, 2013.

[21] K. T. Atanassov, "Intuitionistic fuzzy sets," Fuzzy Sets and Systems, vol. 20, no. 1, pp. 87-96, 1986.

[22] B. Zhu, Z. S. Xu, and M. M. Xia, "Dual hesitant fuzzy sets," Journal of Applied Mathematics, vol. 2012, Article ID 879629, 13 pages, 2012.

[23] E. Szmidt and J. Kacprzyk, "Distances between intuitionistic fuzzy sets," Fuzzy Sets and Systems, vol. 114, no. 3, pp. 505-518, 2000.

[24] W. Q. Wang and X. L. Xin, "Distance measure between intuitionistic fuzzy sets," Pattern Recognition Letters, vol. 26, no. 13, pp. 2063-2069, 2005.

[25] Z. S. Xu and M. M. Xia, "On distance and correlation measures of hesitant fuzzy information," International Journal of Intelligent Systems, vol. 26, no. 5, pp. 410-425, 2011.

[26] Z. S. Xu and M. M. Xia, "Distance and similarity measures for hesitant fuzzy sets," Information Sciences, vol. 181, no. 11, pp. 2128-2138, 2011.

[27] Z. S. Xu and X. L. Zhang, "Hesitant fuzzy multi-attribute decision making based on TOPSIS with incomplete weight information," Knowledge-Based Systems, vol. 52, pp. 53-64, 2013.

[28] E. Szmidt and J. Kacprzyk, "Entropy for intuitionistic fuzzy sets," Fuzzy Sets and Systems, vol. 118, no. 3, pp. 467-477, 2001.

[29] I. K. Vlachos and G. D. Sergiadis, "Intuitionistic fuzzy information-applications to pattern recognition," Pattern Recognition Letters, vol. 28, no. 2, pp. 197-206, 2007.

[30] V. Torra and Y. Narukawa, "On hesitant fuzzy sets and decision," in Proceedings of the IEEE International Conference on Fuzzy Systems, pp. 1378-1382, Jeju Island, Korea, August 2009.

[31] D. Verberg, E. J. Purcell, and S. E. Rigdon, Calculus, Person Education, 9th edition, 2009.

[32] D. Diakoulaki, G. Mavrotas, and L. Papayannakis, "Determining objective weights in multiple criteria problems: the critic method," Computers \& Operations Research, vol. 22, no. 7, pp. 763-770, 1995.
[33] Y. Kim and E.-S. Chung, "Fuzzy VIKOR approach for assessing the vulnerability of the water supply to climate change and variability in South Korea," Applied Mathematical Modelling, vol. 37, no. 22, pp. 9419-9430, 2013.

[34] N. Zhang and G. W. Wei, "Extension of VIKOR method for decision making problem based on hesitant fuzzy set," Applied Mathematical Modelling, vol. 37, no. 7, pp. 4938-4947, 2013.

[35] H.-C. Liu, J.-X. You, X.-Y. You, and M.-M. Shan, "A novel approach for failure mode and effects analysis using combination weighting and fuzzy VIKOR method," Applied Soft Computing Journal, vol. 28, pp. 579-588, 2015.

[36] K. Rezaie, S. S. Ramiyani, S. Nazari-Shirkouhi, and A. Badizadeh, "Evaluating performance of Iranian cement firms using an integrated fuzzy AHP-VIKOR method," Applied Mathematical Modelling, vol. 38, no. 21-22, pp. 5033-5046, 2014.

[37] G.-N. Zhu, J. Hu, J. Qi, C.-C. Gu, and Y.-H. Peng, "An integrated AHP and VIKOR for design concept evaluation based on rough number," Advanced Engineering Informatics, vol. 29, no. 3, pp. 408-418, 2015.

[38] N. P. Feng, Y. S. Tang, Z. L. Peng, and S. F. Wang, "The establishment of people's livelihood project evaluation indicators system based on grounded theory method-statistical methodinformation entropy," Forecasting, vol. 32, no. 6, pp. 66-72, 2013 (Chinese)

[39] C. L. Hwang and K. Yoon, Multiple Decision Making Methods and Applications, Springer, Berlin, Germany, 1981. 


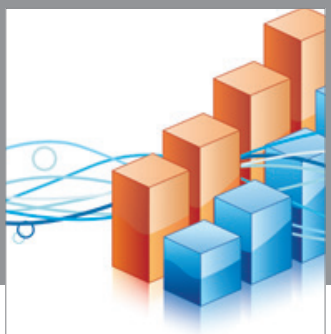

Advances in

Operations Research

vatem alat4

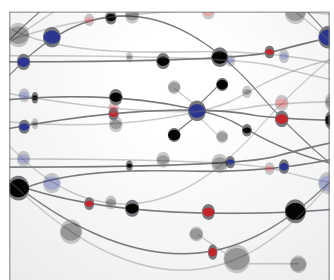

\section{The Scientific} World Journal
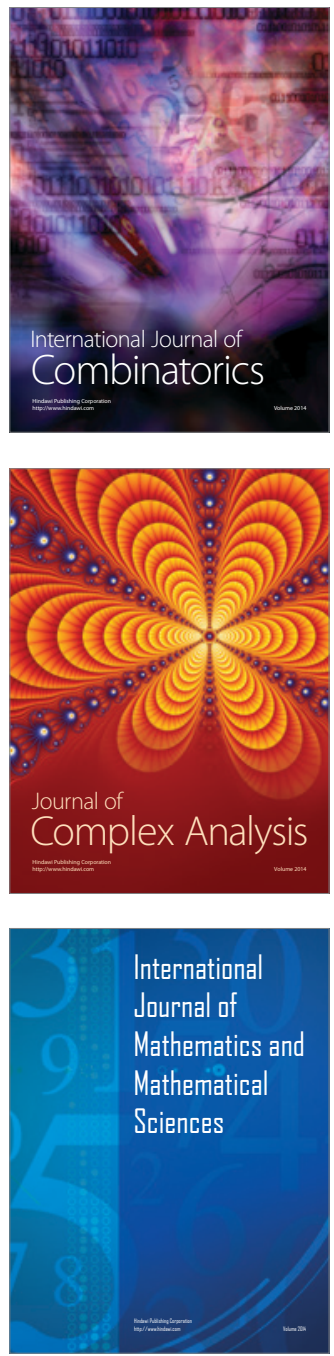
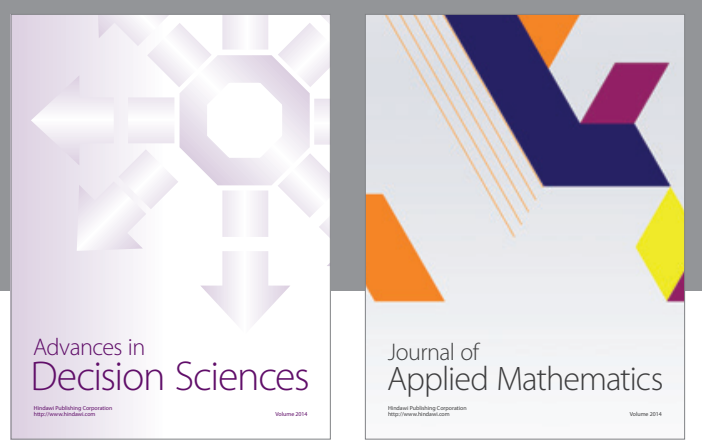

Algebra

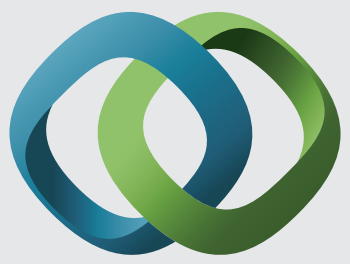

\section{Hindawi}

Submit your manuscripts at

http://www.hindawi.com
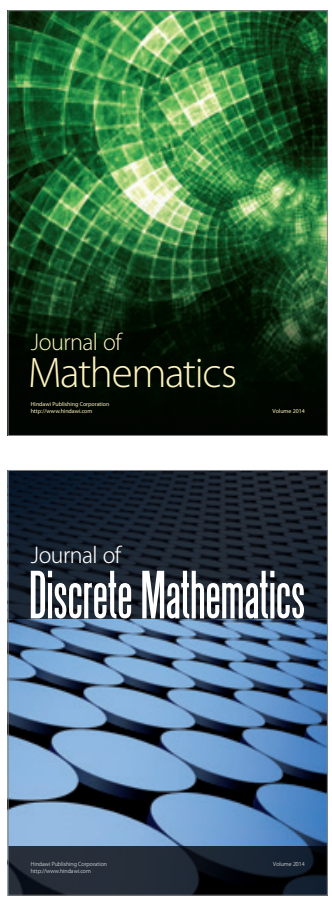

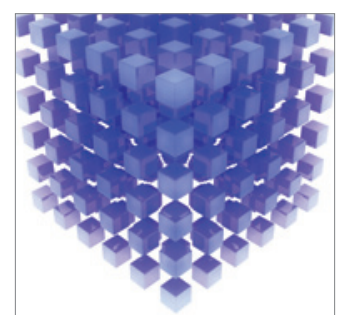

Mathematical Problems in Engineering
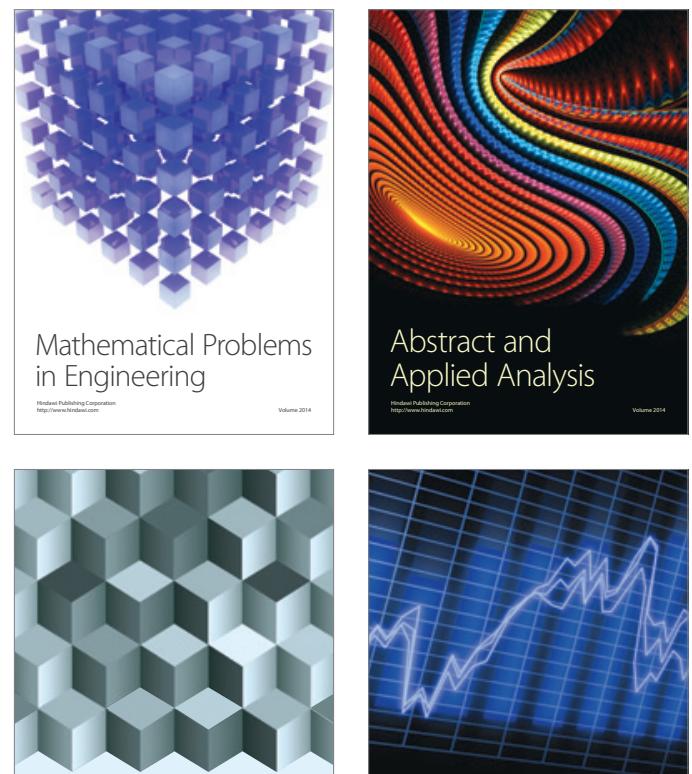

Journal of

Function Spaces

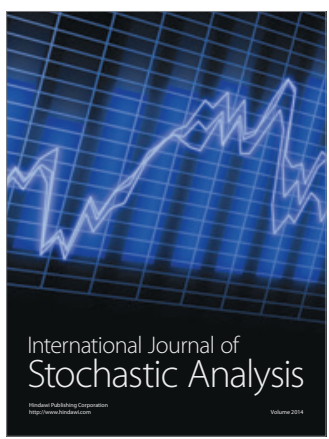

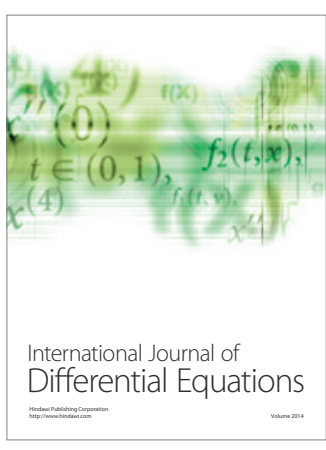
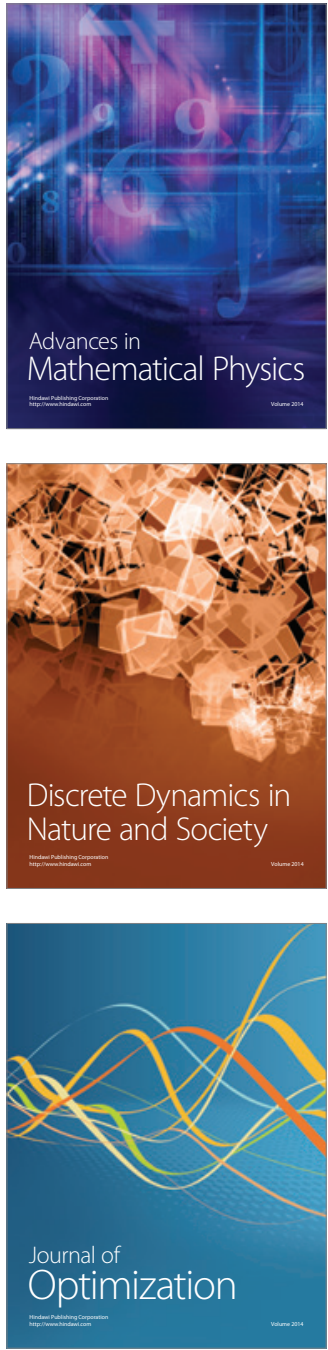